Pacific Northwest

\title{
Materials and Methods for Streamlined Laboratory Analysis of Environmental Samples FY 2016 Report
}

\section{November 2016}

$\begin{array}{ll}\text { RS Addleman } & \text { W Chouyyok } \\ \text { BE Naes } & \text { D Willingham } \\ \text { BK McNamara } & \text { AC Spigner } \\ \text { KB Olsen } & \end{array}$




\title{
DISCLAIMER
}

This report was prepared as an account of work sponsored by an agency of the United States Government. Neither the United States Government nor any agency thereof, nor Battelle Memorial Institute, nor any of their employees, makes any warranty, express or implied, or assumes any legal liability or responsibility for the accuracy, completeness, or usefulness of any information, apparatus, product, or process disclosed, or represents that its use would not infringe privately owned rights. Reference herein to any specific commercial product, process, or service by trade name, trademark, manufacturer, or otherwise does not necessarily constitute or imply its endorsement, recommendation, or favoring by the United States Government or any agency thereof, or Battelle Memorial Institute. The views and opinions of authors expressed herein do not necessarily state or reflect those of the United States Government or any agency thereof.

\author{
PACIFIC NORTHWEST NATIONAL LABORATORY \\ operated by \\ BATTELLE \\ for the \\ UNITED STATES DEPARTMENT OF ENERGY \\ under Contract DE-AC05-76RL01830
}

Printed in the United States of America

Available to $\mathrm{DOE}$ and $\mathrm{DOE}$ contractors from the

Office of Scientific and Technical Information,

P.O. Box 62, Oak Ridge, TN 37831-0062;

ph: (865) 576-8401

fax: $(865) 576-5728$

email: reports $a$ adonis.osti.gov

Available to the public from the National Technical Information Service

5301 Shawnee Rd., Alexandria, VA 22312

ph: (800) 553-NTIS (6847)

email: orders $a$ ntis.gov <http://www.ntis.gov/about/form.aspx $>$

Online ordering: http://www.ntis.gov 


\title{
Materials and Methods for Streamlined Laboratory Analysis of Environmental Samples FY 2016 Report
}

\author{
RS Addleman \\ W Chouyyok \\ BE Naes \\ BK McNamara \\ KB Olsen \\ D Willingham \\ AC Spigner
}

November 2016

Prepared for

the U.S. Department of Energy

under Contract DE-AC05-76RL01830

Pacific Northwest National Laboratory

Richland, Washington 99352 



\section{Summary}

The International Atomic Energy Agency (IAEA) relies upon laboratory analysis of environmental samples (typically referred to as "swipes”) collected during on-site inspections of safeguarded facilities to support the detection and deterrence of undeclared activities. Unfortunately, chemical processing and assay of the samples is slow and expensive. A rapid, effective, and simple extraction process and analysis method is needed to provide certified results with improved timeliness at reduced costs (principally in the form of reduced labor), while maintaining or improving sensitivity and efficacy.

To address these safeguard needs the Pacific Northwest National Laboratory (PNNL) explored and demonstrated improved methods for environmental sample (ES) analysis. Improvements for both bulk and particle analysis were explored. To facilitate continuity and adoption, the new sampling materials and processing methods will be compatible with existing IAEA protocols for ES analysis. PNNL collaborated with Oak Ridge National Laboratory (ORNL), which performed independent validation of the new bulk analysis methods and compared performance to traditional IAEA's Network of Analytical Laboratories (NWAL) protocol.

ORNL efforts are reported separately. This report describes PNNL's FY 2016 progress, which was focused on analytical application supporting environmental monitoring of uranium enrichment plants and nuclear fuel processing. In the future the technology could be applied to other safeguard applications and analytes related to fuel manufacturing, reprocessing, etc. PNNL's FY 2016 efforts were broken into two tasks and a summary of progress, accomplishments and highlights are provided below.

Principal progress and accomplishments on Task 1, Optimize Materials and Methods for ICP-MS Environmental Sample Analysis, are listed below.

- Completed initial procedure for rapid uranium extraction from ES swipes based upon carbonateperoxide chemistry (delivered to ORNL for evaluation).

- Explored improvements to carbonate-peroxide rapid uranium extraction chemistry.

- Evaluated new sampling materials and methods (in collaboration with ORNL).

- Demonstrated successful ES extractions from standard and novel swipes for a wide range uranium compounds of interest including $\mathrm{UO}_{2} \mathrm{~F}_{2}$ and $\mathrm{UO}_{2}\left(\mathrm{NO}_{3}\right)_{2}, \mathrm{U}_{3} \mathrm{O}_{8}$ and uranium ore concentrate.

- Completed initial discussions with commercial suppliers of PTFE swipe materials.

- Submitted one manuscript for publication. Two additional drafts are being prepared.

Principal progress and accomplishments on Task 2, Optimize Materials and Methods for Direct SIMS Environmental Sample Analysis, are listed below.

- Designed a SIMS swipe sample holder that retrofits into existing equipment and provides simple, effective, and rapid mounting of ES samples for direct assay while enabling automation and laboratory integration.

- Identified preferred conductive sampling materials with better performance characteristics.

- Ran samples on the new PNNL NWAL equivalent Cameca 1280 SIMS system. 
- Obtained excellent agreement between isotopic ratios for certified materials and direct SIMS assay of very low levels of $\mathrm{LEU}$ and $\mathrm{HEU} \mathrm{UO}_{2} \mathrm{~F}_{2}$ particles on carbon fiber sampling material. Sample activities range from 1 to 500 CPM (uranium mass on sample is dependent upon specific isotope ratio but is frequently in the subnanogram range).

- Found that the presence of the UF molecular ions, as measured by SIMS, provides chemical information about the particle that is separate from the uranium isotopics and strongly suggests that those particles originated from an $\mathrm{UF}_{6}$ enrichment activity.

- Submitted one manuscript for publication. Another manuscript is in preparation.

Significant advances were made during the FY 2016 efforts but additional focused efforts are needed to finalize the materials and methods needed to provide finished protocols for streamlined analysis with either bulk and particle measurement methods. A discussion of potential future work is found at the end of the annual report. A fact sheet for the program and technology can be found in Appendix A. 


\section{Acknowledgments}

This research was supported by the U.S. National Nuclear Security Administration (NNSA) Office of Nonproliferation and International Security (NA-24), and its Next Generation Safeguards Initiative, under Contract DE-AC05-76RL01830 with the U.S. Department of Energy. Pacific Northwest National Laboratory is a multi-program national laboratory operated by Battelle for the U.S. Department of Energy. 



\section{Acronyms and Abbreviations}

$\begin{array}{ll}\text { amu } & \text { atomic mass unit } \\ \text { cpm } & \text { counts per minute } \\ \text { ES } & \text { environmental sample } \\ \text { FOV } & \text { field of view } \\ \text { FY } & \text { hiscal year } \\ \text { HEU } & \text { International Atomic Energy Agency } \\ \text { IAEA } & \text { inductively coupled plasma mass spectrometry } \\ \text { ICP-MS } & \text { lightly enriched uranium } \\ \text { LEU } & \text { multi-collector inductively coupled plasma mass spectrometer } \\ \text { MC-ICP-MS } & \text { NNSA Office of Research and Development } \\ \text { NA-22 } & \text { National Nuclear Security Administration } \\ \text { NNSA } & \text { Network of Analytical Laboratories } \\ \text { NWAL } & \text { Oak Ridge National Laboratory } \\ \text { ORNL } & \text { Pacific Northwest National Laboratory } \\ \text { PNNL } & \text { polytetrafluoroethylene } \\ \text { PTFE } & \text { research and development } \\ \text { R\&D } & \text { secondary ion mass spectrometry } \\ \text { SIMS } & \text { thermal ionization mass spectrometry } \\ \text { TIMS } & \text { technology readiness level } \\ \text { TRL } & \text { uranium ore concentrate } \\ \text { UOC } & \text { X-ray florescence } \\ \text { XRF } & \end{array}$





\section{Contents}

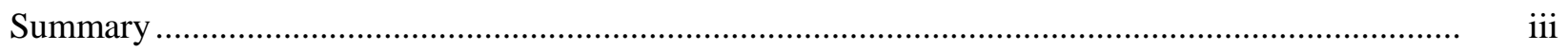

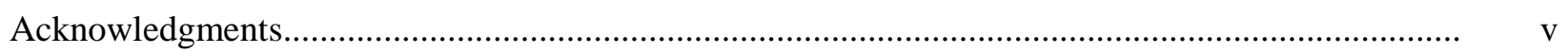

Acronyms and Abbreviations ..................................................................................................... vii

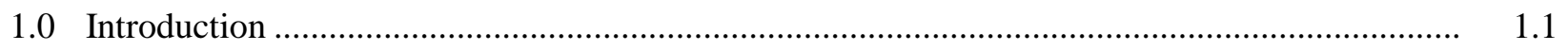

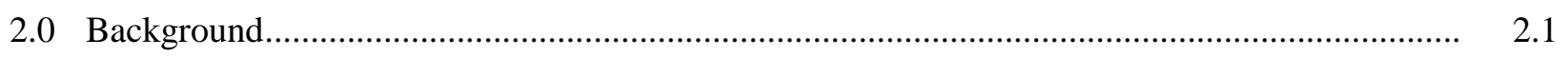

3.0 Task 1. Optimize Materials and Methods for ICP-MS Environmental Sample Analysis ........... 3.1

3.1 Extraction Chemistry for Improved Bulk (ICP-MS) Measurement of Environmental

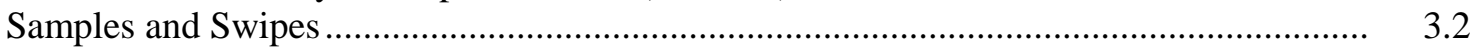

3.1.1 Improved Extraction Chemistry for Environmental Swipe Sample Processing........ 3.4

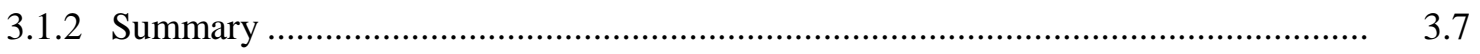

3.2 Evaluation and Validation of New Swipe/Sampling Materials....................................... 3.7

3.2.1 Sampling Materials Selected for Evaluation of Bulk Uranium Extraction .............. 3.8

3.2.2 Evaluation of Selected Bulk Environmental Sampling Materials ............................ 3.10

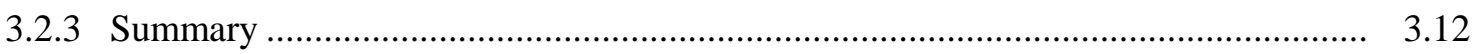

4.0 Task 2. Optimize Materials and Methods for Direct SIMS Environmental Sample Analysis ..... $\quad 4.1$

4.1 SIMS Environmental Sample Methods Development ......................................................

4.1.1 Improved Sample Mounting for Direct Assay of Swipe Sampling Cloth................ 4.3

4.2 Direct Swipe Sample Analysis with SIMS...................................................................... 4.5

4.2.1 Validation of Enrichment Activities with a Chemical Signature from SIMS

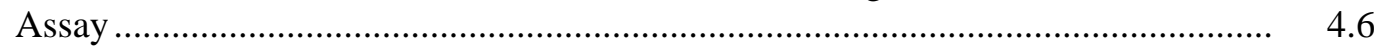

4.3 Conductive Fabric Sampling/Materials to Enable Direct SIMS Assay ….......................... 4.7

4.3.1 CNT Fabric and Graphene Coating Materials..................................................... 4.9

4.3.2 Carbon Fiber--Carbon Twill Weaves .................................................................. 4.10

4.3.3 Graphite Fabric................................................................................................ 4.10

4.3.4 Metal-coated Fabric................................................................................. 4.10

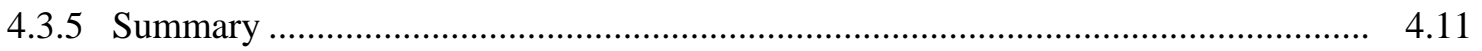

5.0 Task 3. Independent Technology Evaluation and Validation................................................. 5.1

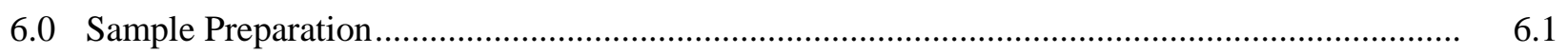

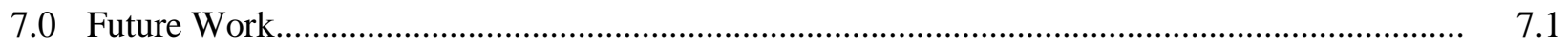



Appendix A Safeguards Technology Fact Sheet, Streamlined Analysis of Environmental Samples... A.1

Appendix B Extraction Method Details for Bulk Environmental Sample Swipes ............................ B.. B

Appendix C Measurement Methods for Potential SIMS Sampling Materials .................................... C.1

Appendix D High Magnification Images of Potential Sampling Fabrics that May Enable Direct SIMS

D.1 


\section{Figures}

3.1. A Conceptual Graphic Showing the Steps in Existing and Proposed Methods for Preparation and Analysis of Bulk Samples with ICP-MS......

3.2. Samples of the Standard and Promising Environmental Sampling Materials. ........................... 3.8

3.3. Different Carbon Fiber Materials.......................................................................................... 3.10

4.1. Comparison of the Existing Technique and the New Approach for Preparation and Analysis with SIMS for Particle Assay.

4.2. A Schematic for Sample Mount that Enables Direct Assay of (conductive) Environmental Swipe Samples with SIMS.

4.3. SIMS Images for a Single Field of View on a Carbon Fiber Cloth Sample that had been Used to Collect Dust from a surface that had Low Levels of $\mathrm{UF}_{6}$ Particles.

6.1. Experimental Apparatus for Deposition of $\mathrm{UF}_{6}$ onto Coupons.

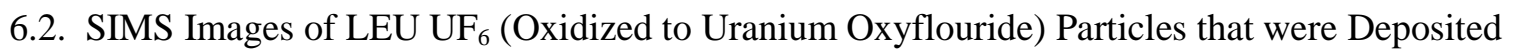
Directly upon Carbon Fiber Sampling Cloth.

6.3. An Example of Dust Samples on Carbon Fiber Sampling Cloth.

\section{Tables}

3.1. Comparative Extraction Chemistries

3.2. Impact of Ammonium Carbonate Concentration on Extraction Efficiency ${ }^{(a)}$ (with $2 \mathrm{M} \mathrm{H}_{2} \mathrm{O}_{2}$ ) ...

3.3. Impact of Hydrogen Peroxide Concentration on Extraction Efficiency ${ }^{(\mathrm{a})}$ with $\left(\mathrm{NH}_{4}\right)_{2} \mathrm{CO}_{3} \ldots \ldots \ldots . . .3 .5$

3.4. Impact of Hydrogen Peroxide Concentration on Extraction Efficiency ${ }^{(\mathrm{a})}$ with $\mathrm{Na}_{2} \mathrm{CO}_{3} \ldots \ldots \ldots \ldots \ldots . . . . . .3 .6$

3.5. Extraction Performance ${ }^{(a)}$ from Different Swipe materials for Preferred Chemical Conditions... $\quad 3.7$

3.6. Background and Extraction Efficiency for Uranium Compounds from Selected Sampling Materials with Selected Extraction Chemistries

4.1. Comparative SIMS Results for Enriched $\mathrm{UF}_{6}$ Samples $^{(\mathrm{a})}$ on Carbon Fiber Sampling Material ... $\quad 4.5$

4.2. Properties of Selected Sampling and Reference Materials 


\subsection{Introduction}

The International Atomic Energy Agency (IAEA) relies upon laboratory analysis of environmental samples (typically "swipes" from surfaces of interest) during on-site inspections of safeguarded facilities to support the detection and deterrence of undeclared activities. Unfortunately, using today's methods chemical processing and assay of the swipe samples is slow, which can significantly delay reporting of results and lead to large sample backlogs. Faster, simpler sample processing and analysis methods are needed to provide certified results with improved timeliness and at reduced cost, while maintaining or improving sensitivity and efficacy.

This effort builds upon a previous National Nuclear Security Administration (NNSA) Office of Research and Development (NA-22) investment "Improved Collection and Measurement of Safeguards Signatures Using Engineered Materials and Processes,” an FY 2013-2015 program, which explored potential options and technologies. The NA-22 project advanced the technology readiness level (TRL) from 2 to 3-4. The project explored and demonstrated the means to enable significant improvements in analytical efficiency for the primary analytical methods used by the IAEA (inductively coupled plasma mass spectrometry and secondary ion mass spectrometry for environmental sample (ES) analysis. Alternative sampling materials and improved analytical methods were explored in the present effort. To facilitate continuity and adoption the new sampling materials and processing methods were selected to be completely compatible with existing IAEA protocols and equipment for ES analysis.

This present International Nuclear Safeguards program (NA-241) effort directly addresses the recognized need for greater efficiency in the processing of environmental samples by the IAEA, and indirectly supports the IAEA's desire for enhanced effectiveness in environmental sample analysis (e.g., high-priority capability needs 10.2 and 10.3 of the IAEA's Long Term R\&D Plan). FY 2016 work was focused on analytical application supporting environmental monitoring of uranium enrichment plants and nuclear fuel processing. While FY 2016 efforts focus of $\mathrm{UF}_{6}$ measurement, the methods were also successfully applied to uranium oxides, nitrates, and natural minerals. Future work could apply the new methods to other analytes such as plutonium and trace isotopes of forensic interest. Consequently, the developed and mature technology could support monitoring of reprocessing activities as well as other ES safeguards needs in the future. A fact sheet for the program and technology can be found in Appendix A.

FY 2016 work was divided into three tasks (and this report is organized by these tasks).

\section{Task 1. Optimize Materials and Methods for ICP-MS Environmental Sample Analysis}

Inductively coupled plasma mass spectrometry (ICP-MS) assay of digested swipe samples is the core method for ES assay. The task is developing and demonstrating sampling materials and extraction methods that provide faster, simpler, more cost-effective bulk assay of environmental samples. We will move beyond previous proof-of-principal efforts by maturing the technology and integrating the material and extraction methods into a coherent new protocol. Work on this task is described in Section 3.

\section{Task 2: Optimize Materials and Methods for Direct SIMS Environmental Sample Analysis}

Secondary ion mass spectrometry (SIMS) is the principle method for particle analysis in ES swipes. This effort will develop faster, cheaper, and more automated SIMS technology to addresses its major analytical shortcomings. The effort will develop defined analysis methods and commercial sources of 
sampling materials that enable direct SIMS assay of surface swipe samples. The new process will accelerate and simplify the existing SIMS process as well as increase the throughput and utility of existing SIMS instruments. Current efforts are aimed at developing methods that provide direct assay (minimal sample preparation) and automated searching for particles of interests on ES swipes (and subsequent isotopic assay). Work on this task is described in Section 4.

\section{Task 3. Independent Technology Evaluation and Validation}

Oak Ridge National Laboratory (ORNL) evaluated the materials and procedures generated at Pacific Northwest National Laboratory (PNNL) for bulk ES assay and independently assessed them against standard Network of Analytical Laboratories (NWAL) protocols using ICP-MS. ORNL analysis provided validation of the strengths and limitations of the new materials and methods, and input for further optimization the new procedures. With this data a comparison on the finalized protocols can then be made to gauge their advantages versus the standard analytical methods relative to accuracy, precision, cost and timeliness. Work on this task is described in Section 5. 


\subsection{Background}

Environmental sampling is a fundamental component of the International Atomic Energy Agency's (IAEA's) Safeguards regime. There is a need for faster, simpler, cheaper analytical techniques for processing environmental swipe samples at the IAEA and its supporting Network of Analytical Laboratories. For decades laboratory instrumentation such as mass spectrometers have undergone sustained development efforts to improve detection limits. However, the materials for sampling and analytical methods used for processing have not changed since first implemented by the IAEA in the early 1990s. This project explored the development of improved procedures new sampling materials and subsequent processing methods to provide better analysis of environmental safeguard samples. Currently, samples are taken with natural fiber materials (e.g., cotton), which lack chemical homogeneity and physical and chemical stability, have an unstable background, and require extensive processing for sample analysis. Better sampling materials and associated processing methods should benefit Safeguards efforts by reducing sample preparation cost and time, improving analytical performance, and increasing laboratory throughput.

This effort builds upon a previous NNSA NA-22 investment "Improved Collection and Measurement of Safeguards Signatures Using Engineered Materials and Processes”, an FY 2013-2015 program that explored potential options and technologies. The focus of this previous program was to explore the means to enable significant improvements in analytical efficiency for the primary analytical methods used by the IAEA (ICP-MS and SIMS) for environmental-sample analysis. Understanding the various materials that have previously been used and assessing novel materials that might be provided a rational basis for selecting promising candidates for subsequent development efforts. A large number of potential sampling materials were reviewed and evaluated. The baseline for comparison was the IAEA's standard muslin sampling material along with its standard associated sample processing and assay methods.

Teflon (polytetrafluoroethylene) coated fabric and carbon fiber cloth were found to be very promising materials. These chemically inert materials had low background, were compatible with existing IAEA methods, and did not require the total digestion of the swipe material to recover the uranium. These findings simplify, accelerate, and reduce the cost of bulk environmental sample analysis. For processing of bulk samples a simple, fast, nontoxic, single stage (“one-pot”) carbonate extraction process was demonstrated to provide effective extraction and allow subsequent (ICP-MS) analysis method. An X-ray fluorescence (XRF) sampling screening method that used the rapid extraction methods and the new sampling materials were developed and demonstrated to enable rapid, simple sample triage and assay with nanogram-level sensitivity for uranium. Additional work is needed to optimize the process and make it an NWAL-compatible protocol.

Some carbon fiber fabrics were found to provide sufficient conductivity to enable direct SIMS analysis of the sample. These conductive carbon fiber fabrics were shown to enable direct particulate analysis of samples without any of the extensive sample preparation traditionally required for the current swipe media. It was demonstrated that individual enriched particles could be found directly on the new carbon fiber sampling materials and accurate isotopic ratios could be obtained with SIMS. Additional work is needed to optimize the materials and methods to provide a viable NWAL-compatible protocol.

A full summary of the previous work can be found in the final NA-22 program report by Addleman et al. 2016, Improved Collection and Measurement of Safeguards Signatures Using Engineered Material. 



\subsection{Task 1. Optimize Materials and Methods for ICP-MS Environmental Sample Analysis}

ICP-MS assay of digested swipe samples is the core method for ES assay. Task 1 was focused on developing and demonstrating sampling materials and extraction methods that provide faster, simpler, and more cost-effective bulk assay of environmental samples. The research team moved beyond previous proof-of-principal efforts (supported by NA-22) and matured the technology and integrated the material and extraction methods into a validated coherent new protocol. Figure 3.1 shows a conceptual graphic of the existing and proposed new methods for preparation and analysis of bulk samples with ICP-MS.

Existing Protocol

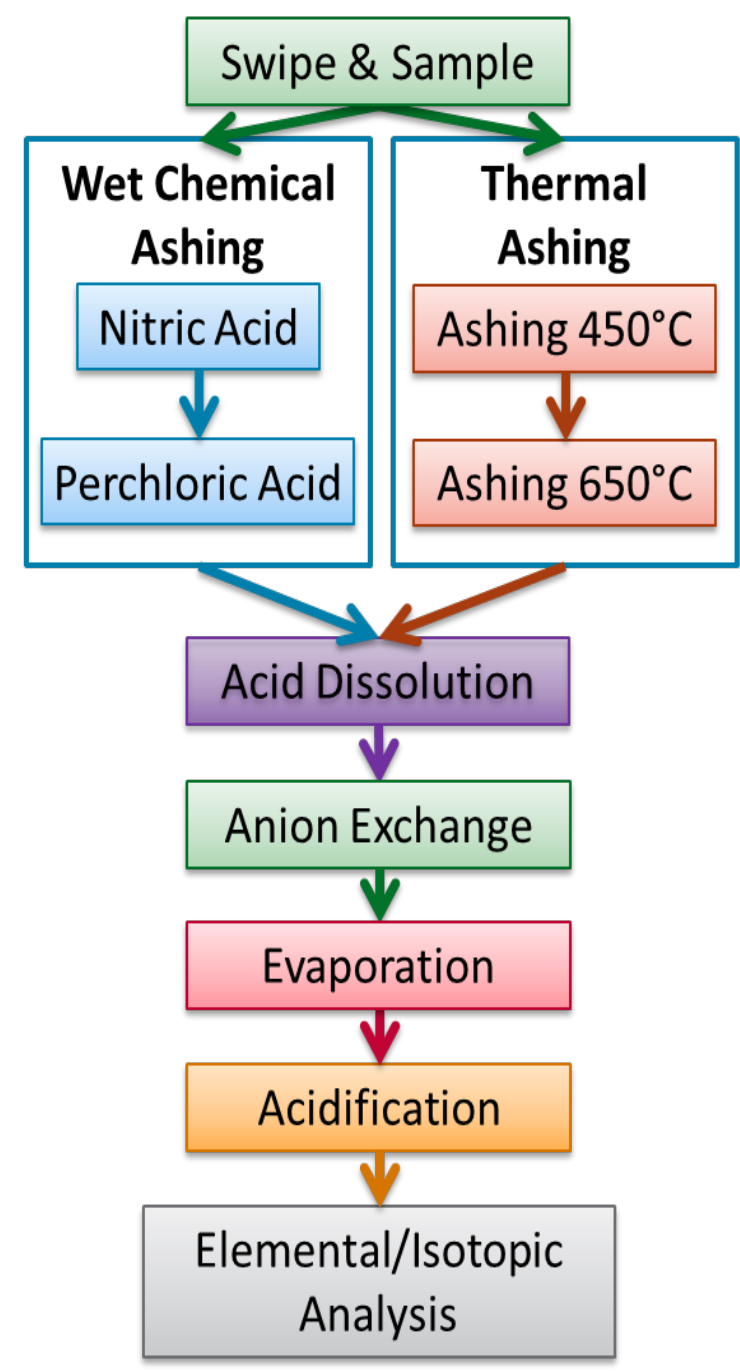

New Approach

\section{Swipe \& Sample}



Figure 3.1. A Conceptual Graphic Showing the Steps in Existing and Proposed Methods for Preparation and Analysis of Bulk Samples with ICP-MS. The methods and materials under development provide simpler, faster, and more cost effective methods for measurement of safeguard environmental samples. 
The focus of Task 1 is optimizing materials and methods for improved bulk environmental samples/surface swipe analysis. The task had two primary efforts with specific requirements that are summarized below.

- Development of Rapid Extraction Chemistry

- Avoid digestion of swipe

- Simple fast preparation for ICP-MS assay

- Works with all swipe materials

- Validation of New Swipe/Sampling Material

- Inert in acid or base digestion processes

- Ultralow stable background

- Commercially available

- Nonreactive with enrichment samples

- Conductivity for SIMS preferred

FY 2016 results for these efforts are detailed subsequently in Sections 3.1 and 3.2 and are summarized below.

- Completed initial procedure for rapid uranium extraction from ES swipes based upon carbonateperoxide chemistry (delivered to ORNL for evaluation).

- Explored improvements to carbonate-peroxide rapid uranium extraction chemistry.

- Evaluation of new sampling materials and methods (in collaboration with ORNL).

- Demonstrated successful ES extractions from standard and novel swipes for a wide range uranium compounds of interest including $\mathrm{UO}_{2} \mathrm{~F}_{2}$ and $\mathrm{UO}_{2}\left(\mathrm{NO}_{3}\right)_{2}, \mathrm{U}_{3} \mathrm{O}_{8}$ and uranium ore concentrate.

- Completed initial discussions with commercial suppliers of polytetrafluoroethylene (PTFE) swipe materials have been successfully completed.

- Completed one manuscript for publication and prepared two manuscript drafts.

- Prepared final report containing FY 2016 results for efforts on Task 1.

\subsection{Extraction Chemistry for Improved Bulk (ICP-MS) Measurement of Environmental Samples and Swipes}

ICP-MS requires extracting and dissolving the analyte from the surface sampling materials (swipes) before analysis. The extraction of signatures from muslin swipes and subsequent chemical processing of the sample to get it into a measurable form is a time-consuming, multistep process. Extraction and sample preparation are major factors limiting IAEA NWAL throughput. Consequently, development and demonstration of a fast, efficient, simple extraction processes for swipe materials that are compatible with subsequent ICP-MS analysis is a major focus area for the program. 
The FY 2016 effort to improve the extraction chemistry of bulk environmental sample processing before subsequent ICP-MS analysis focused on optimizing and validating the carbonate-peroxide chemistry. As shown in Table 3.1 for a challenging uranium compound of IAEA interest $\left(\mathrm{U}_{3} \mathrm{O}_{8}\right)$ carbonate-peroxide chemistry is better than most methods and as good as accepted extraction methods (at the $2 \mathrm{M}$ carbonate level). The carbonate-peroxide chemistry provides simple overnight uranium extraction, does not requires digestion of the swipe-associated increase in background, time and processing steps. Unlike the present NWAL methods that digest the entire sample and mobilize all metals, the carbonate extraction chemistry should also have the advantage of reducing or eliminating the extraction of materials that might become interferents (i.e., $\mathrm{Pb}, \mathrm{Pt}, \mathrm{W}, \mathrm{Bi}$, and $\mathrm{Hg}$ ) during the ICP-MS measurement.

Table 3.1. Comparative Extraction Chemistries

\begin{tabular}{l|c}
\hline \multicolumn{1}{c|}{ Extraction Chemistry } & $\begin{array}{c}\text { \% Extraction }{ }^{(a)} \text { of } \mathrm{U}_{3} \mathrm{O}_{8} \text { from } \\
\text { Muslin }\end{array}$ \\
\hline DI Water & $3( \pm 1)$ \\
$0.0001 \mathrm{M} \mathrm{HNO}_{3}$ & $1( \pm 0.7)$ \\
$0.01 \mathrm{M} \mathrm{Na}_{2} \mathrm{CO}_{3}$ & $15( \pm 0.3)$ \\
$0.01 \mathrm{M} \mathrm{HNO}_{3}$ & $47( \pm 3)$ \\
$6.0 \mathrm{M} \mathrm{HNO}_{3}$ & $56( \pm 5)$ \\
Standard Method ${ }^{(b)}$ & $100( \pm 1)$ \\
\hline $0.02 \mathrm{M} \mathrm{Na}_{2} \mathrm{CO}_{3} / \mathrm{NaHCO}_{3}$ & $11( \pm 1.4)$ \\
$1.0 \mathrm{M} \mathrm{Na}_{2} \mathrm{CO}_{3}$ & $58( \pm 3)$ \\
$1 \mathrm{M}\left(\mathrm{NH}_{4}\right)_{2} \mathrm{CO}_{3} / \mathrm{H}_{2} \mathrm{O}_{2}$ & $92( \pm 14)$ \\
$2 \mathrm{M}\left(\mathrm{NH}_{4}\right)_{2} \mathrm{CO}_{3} / \mathrm{H}_{2} \mathrm{O}_{2}$ & $98( \pm 4)$ \\
\hline (a) $18 \mathrm{~h} \mathrm{contact} \mathrm{time,} \mathrm{shaken,} \mathrm{triplicate} \mathrm{runs}$ \\
(b) Vogt et al. 2001 , Kohler et. al., Environ. Sci. Technol. 2004, \\
Smith et. al., J Radioanal Nucl Chem 2009 \\
\hline
\end{tabular}

Specific FY 2016 efforts in extraction chemistry included:

- Exploring better carbonate and peroxide concentrations

- Performing compatibility studies with the different swipe materials

- Developing initial draft analytical protocol/procedure and transferring it to ORNL for evaluation

- Packaging information to report on research in peer-reviewed literature.

With the intent to make the efforts of the program available to the user community, and benefit from the external peer review process, the research is being prepared for publication. Efforts included:

- Completion and submission of a manuscript, "Rapid Extraction and Assay of Uranium from Environmental Surface Sampling Material,” the work that began under NA-22 support.

- Drafting of “Review and Assessment of Surface Sampling Materials for Nuclear Applications,” likely with ORNL.

- Drafting a manuscript, "PTFE Polymer-coated Fabric Materials for Improved International Nuclear Safeguard Sampling and Analysis Efforts.” 
A fourth publication maybe assembled collaboratively with ORNL when the extraction chemistry is finalized.

Initial PNNL efforts were completed on the rapid carbonate-peroxide uranium extraction chemistry and an initial procedure/protocol for rapid chemical extraction processing was developed and delivered to ORNL. ORNL determined:

- The carbonate-peroxide uranium extraction chemistry was effective and efficient for uranium for a range of swipe materials and provided a low background.

- For repeated use on the NWAL style multi-collector (MC) ICP-MS, equipped with a high efficiency desolvating nebulizer (a "dry" nebulizer), the research team has to reduce the solids (carbonate) in the final processed sample to avoid plugging of a desolvating nebulizer coating the MC plates and degrading instrument performance. This unanticipated problem resulted in significant efforts to reduce carbonate concentration while maintaining good extraction efficiency.

- Even with higher carbonate concentrations a modified procedure rapid sensitive uranium analysis is still possible. The carbonate leachate is simply evaporated and the carbonate residue ignited and reduced in a furnace. Similar to portions of the existing NWAL method, the residue is then simply acidified, cleaned up with microcolumn, and run on ICP-MS with good results. The significant difference is the reduction in time required for the procedure: from 15 days for the standard ORNL $N W A L$ procedure to digest and chemically process a swipe sample for uranium to three days for the new procedure.

\subsubsection{Improved Extraction Chemistry for Environmental Swipe Sample Processing}

Optimization of carbonate-peroxide extractions chemistries was an important part of work planned for the FY 2016 efforts. FY 2016 ORNL results showing that the 2M carbonate concentration (previously shown to be effective) plugged the nebulizer, coated the MC plates, and degraded ICP-MS performance. This required unanticipated efforts to find effective carbonate-peroxide extraction conditions and methods at lower reactant concentrations. These efforts are summarized below. Experimental details for the extraction can be found in Appendix B. In context with subsequent discussions and data tables muslin is the cotton-based IAEA standard swipe material TexWipe TX304.

$\mathrm{U}_{3} \mathrm{O}_{8}$ and $\mathrm{UO}_{2} \mathrm{~F}_{2}$ particles were selected for optimization studies of carbonate-peroxide extraction chemistry since these materials of interest have very different chemical properties. $\mathrm{U}_{3} \mathrm{O}_{8}$ particles are associated with oxides from fuel processing and are chemically hard to extract and mobilize. $\mathrm{UO}_{2} \mathrm{~F}_{2}$ particles are associated with aged enrichment samples and are chemically reactive making them easy to extract and mobilize. Optimization studies of the carbonate-peroxide extraction chemistry studies are shown in Tables 3.2-3.5. Table 3.2 shows the impact of ammonium carbonate concentration on uranium extraction (with $2 \mathrm{M} \mathrm{H}_{2} \mathrm{O}_{2}$ ).

In Table 3.2 nearly complete extraction of $\mathrm{U}_{3} \mathrm{O}_{8}$ can be seen at $0.2-2.0 \mathrm{M}\left(\mathrm{NH}_{4}\right)_{2} \mathrm{CO}_{3}$, while complete extraction of $\mathrm{UO}_{2} \mathrm{~F}_{2}$ can be seen at concentrations as low as $0.002 \mathrm{M}$. Although a concentration of $0.6 \mathrm{M}$ $\left(\mathrm{NH}_{4}\right)_{2} \mathrm{CO}_{3}$ provided excellent extraction efficiency, it is still considered a high carbonate concentration and the plugging of a desolvating nebulizer are unlikely to be eliminated. Concentrations of $0.02 \mathrm{M}$ and $0.2 \mathrm{M}\left(\mathrm{NH}_{4}\right)_{2} \mathrm{CO}_{3}$ provided good extraction efficiency; therefore, they were selected for further study the 
optimization of the carbonate-peroxide. Extraction values similar to those shown for muslin in Table 3.2 were observed for PTFE coated swipes and similar results are expect for carbon fibers swipes (depending upon the typical of carbon fiber used and its chemical pretreatment). Extraction measurement variance can be higher with PTFE and carbon fiber sampling materials due issues with wetting of the chemical inert hydrophobic surfaces if the experimental configuration and sample handling are not appropriate and effective. This statement is applicable to interpretation of data from Tables 3.1-3.16.

Table 3.2. Impact of Ammonium Carbonate Concentration on Extraction Efficiency ${ }^{(a)}$ (with $2 \mathrm{M} \mathrm{H}_{2} \mathrm{O}_{2}$ )

\begin{tabular}{l|ll}
\hline \multicolumn{1}{c}{$\begin{array}{c}\left(\mathrm{NH}_{4}\right) 2 \mathrm{CO}_{3} \\
\text { Concentration }(\mathrm{M})\end{array}$} & \multicolumn{2}{c}{ \% Extraction from Muslin } \\
\cline { 2 - 3 } & $\mathrm{U}_{3} \mathrm{O}_{8}$ & $\mathrm{UO}_{2} \mathrm{~F}_{2}$ \\
\hline 0.002 & $79( \pm 13)$ & $100( \pm 6)$ \\
0.02 & $85( \pm 10)$ & $100( \pm 7)$ \\
0.2 & $91( \pm 8)$ & $100( \pm 5)$ \\
0.6 & $99( \pm 10)$ & $100( \pm 7)$ \\
1 & $98( \pm 4)$ & $100( \pm 5)$ \\
2 & $98( \pm 4)$ & $97( \pm 4)$ \\
\hline
\end{tabular}

(a) Average extraction percentage calculated from triplicate samples, and swipes contacted were treated within extraction solutions for 18 hours with continued shaking at $100 \mathrm{rpm}$.

Table 3.3 shows the impact of the concentration of $\mathrm{H}_{2} \mathrm{O}_{2}$ on extraction efficiency for $0.02 \mathrm{M}$ and $0.2 \mathrm{M}\left(\mathrm{NH}_{4}\right)_{2} \mathrm{CO}_{3}$. The reactive compound $\mathrm{UO}_{2} \mathrm{~F}_{2}$ can be observed to quantitatively extracted (for 0.02 and $0.2 \mathrm{M}\left(\mathrm{NH}_{4}\right)_{2} \mathrm{CO}_{3}$ ) with peroxide concentrations at or above $2 \mathrm{M}$. The increased concentration of $\mathrm{H}_{2} \mathrm{O}_{2}$ significantly and positively affects the extraction of $\mathrm{U}_{3} \mathrm{O}_{8}$. The improvement in extraction efficiency of $\mathrm{U}_{3} \mathrm{O}_{8}$ with increasing the $\mathrm{H}_{2} \mathrm{O}_{2}$ concentration is not surprising, given need to break down and oxidize the relatively nonreactive $\mathrm{U}_{3} \mathrm{O}_{8}$. The data in Table 3.3 demonstrate that the complete extraction of uranium particles can be obtained from the low $(0.02 \mathrm{M})$ concentrations of $\left(\mathrm{NH}_{4}\right)_{2} \mathrm{CO}_{3}$ in cooperation with high concentration of $\mathrm{H}_{2} \mathrm{O}_{2}(4 \mathrm{M})$. However, high concentration of $\mathrm{H}_{2} \mathrm{O}_{2}$ may cause other problems for the fast analysis - the higher the concentration of $\mathrm{H}_{2} \mathrm{O}_{2}$ the more bubbles are produced in the extractant solution, resulting off-gassing issues that have to be addressed.

Extraction values similar to those shown for muslin in Table 3.3 were observed for PTFE-coated swipes and similar trends are expect for carbon fibers swipes (depending upon the type of carbon fiber used and its chemical pretreatment).

Table 3.3. Impact of Hydrogen Peroxide Concentration on Extraction Efficiency ${ }^{(\mathrm{a})}$ with $\left(\mathrm{NH}_{4}\right)_{2} \mathrm{CO}_{3}$

\begin{tabular}{l|ll|ll}
\hline \multirow{2}{*}{$\mathrm{H}_{2} \mathrm{O}_{2}$ Concentration (M) } & \multicolumn{2}{|c|}{ \% Extraction $\mathrm{U}_{3} \mathrm{O}_{8}$ from Muslin } & \multicolumn{2}{c}{ \% Extraction $\mathrm{UO}_{2} \mathrm{~F}_{2}$ from Muslin } \\
\cline { 2 - 5 } & $0.02 \mathrm{M}\left(\mathrm{NH}_{4}\right)_{2} \mathrm{CO}_{3}$ & $0.2 \mathrm{M}\left(\mathrm{NH}_{4}\right)_{2} \mathrm{CO}_{3}$ & $0.02 \mathrm{M}\left(\mathrm{NH}_{4}\right)_{2} \mathrm{CO}_{3}$ & $0.2 \mathrm{M}_{\left(\mathrm{NH}_{4}\right)_{2} \mathrm{CO}_{3}}$ \\
\hline 1 & $47( \pm 6)$ & $30( \pm 2)$ & $75( \pm 8)$ & $98( \pm 5)$ \\
2 & $85( \pm 10)$ & $91( \pm 8)$ & $100( \pm 7)$ & $100( \pm 5)$ \\
3 & $\mathrm{NA}$ & $100( \pm 5)$ & $100( \pm 5)$ & $100( \pm 5)$ \\
4 & $100( \pm 1)$ & $100( \pm 8)$ & $100( \pm 1)$ & $99( \pm 2)$ \\
\hline
\end{tabular}

(a) Average extraction percentage calculated from triplicate samples, and swipes contacted were treated within extraction solutions for 18 hours with continued shaking at $100 \mathrm{rpm}$. 
Other carbonate complexes may be more effective than $\left(\mathrm{NH}_{4}\right)_{2} \mathrm{CO}_{3}$ and were considered as alternative reactants to improve the process. Table 3.4 shows the impact of the concentration of $\mathrm{H}_{2} \mathrm{O}_{2}$ on extraction efficiency for $0.02 \mathrm{M}$ and $0.2 \mathrm{M}$ with $\mathrm{Na}_{2} \mathrm{CO}_{3}$ as a carbonate source. Similar to results with $\left(\mathrm{NH}_{4}\right)_{2} \mathrm{CO}_{3}$ the reactive compound $\mathrm{UO}_{2} \mathrm{~F}_{2}$ can be observed to quantitatively extracted (for 0.02 and $0.2 \mathrm{M} \mathrm{Na}_{2} \mathrm{CO}_{3}$ ) with peroxide concentrations at or above $2 \mathrm{M}$. Extraction values similar to those shown for muslin in Table 3.4 were observed for PTFE-coated swipes and similar trends are expect for carbon fibers swipes (depending upon the type of carbon fiber used and its chemical pretreatment).

Table 3.4. Impact of Hydrogen Peroxide Concentration on Extraction Efficiency ${ }^{(a)}$ with $\mathrm{Na}_{2} \mathrm{CO}_{3}$

\begin{tabular}{l|ll|ll}
\hline \multirow{2}{*}{\begin{tabular}{l}
$\mathrm{H}_{2} \mathrm{O}_{2}$ Concentration \\
\cline { 2 - 5 }
\end{tabular}} & \multicolumn{2}{|c|}{ \% Extraction $\mathrm{U}_{3} \mathrm{O}_{8}$ from Muslin } & \multicolumn{2}{c}{ \% Extraction $\mathrm{UO}_{2} \mathrm{~F}_{2}$ from Muslin } \\
\cline { 2 - 5 } & $0.02 \mathrm{M} \mathrm{Na}_{2} \mathrm{CO}_{3}$ & $0.2 \mathrm{M} \mathrm{Na}_{2} \mathrm{CO}_{3}$ & $0.02 \mathrm{M} \mathrm{Na}_{2} \mathrm{CO}_{3}$ & $0.2 \mathrm{M} \mathrm{Na}_{2} \mathrm{CO}_{3}$ \\
\hline 1 & $80( \pm 3)$ & $100( \pm 4)$ & $76( \pm 2)$ & $100( \pm 1)$ \\
2 & $99( \pm 12)$ & $100( \pm 6)$ & $93( \pm 3)$ & $100( \pm 5)$ \\
3 & $90( \pm 9)$ & $100( \pm 3)$ & $96( \pm 1)$ & $100( \pm 2)$ \\
4 & $84( \pm 9)$ & $100( \pm 2)$ & $100( \pm 2)$ & $100( \pm 4)$ \\
\hline
\end{tabular}

(a) Average extraction percentage calculated from triplicate samples, and swipes contacted were treated within extraction solutions for 18 hours with continued shaking at $100 \mathrm{rpm}$.

Increasing concentration of $\mathrm{H}_{2} \mathrm{O}_{2}$ positively affects the extraction of $\mathrm{U}_{3} \mathrm{O}_{8}$ using $\mathrm{Na}_{2} \mathrm{CO}_{3}$ as a carbonate source (for the same reasons discussed in context with Table 3.3). However $\mathrm{Na}_{2} \mathrm{CO}_{3}$ seems to provide better performance (when compared to $\left(\mathrm{NH}_{4}\right)_{2} \mathrm{CO}_{3}$ for the extraction of $\mathrm{U}_{3} \mathrm{O}_{8}$ at the lower peroxide concentrations). This result suggests $\mathrm{Na}_{2} \mathrm{CO}_{3}$ maybe a promising alternative source of carbonate for carbonate-peroxide extraction process. However, this condition needs to be tested and confirmed with MC-ICP-MS coupled with a "dry" nebulizer (all experiments in this work were analyzed with MC-ICP-MS equipped with nebulizer that can operate with high dissolved solid content). Unlike the ammonia, which can be evaporated out of the sample, the sodium remains in solution. The high concentration of sodium adds to the total solids and may cause issues with the analysis of uranium by ICP-MS (unless there are some limited clean up steps after extraction, as suggested by ORNL in Section 5). Further study is needed regarding the relative advantages of $\mathrm{Na}_{2} \mathrm{CO}_{3}$ and other carbonate sources.

Table 3.5 shows effective extractions for both hard (e.g., $\mathrm{U}_{3} \mathrm{O}_{8)}$ and soft (e.g., $\mathrm{UO}_{2} \mathrm{~F}_{2}$ ) uranium particles of safeguards interest for the carbonate-peroxide chemical conditions given. Clearly, overnight (18-hour) extractions are effective with either the standard muslin (TexSwipe 304) swipes or PTFE sampling material. Similar performance can be expected for carbon fibers swipes (depending upon the carbon fiber used and its chemical pretreatment). Presently, extraction performance becomes nonquantitative (less than 100 percent) for carbonate-peroxide chemical concentrations less than shown. Additional studies are needed to determine if effective and quantitative extraction can be achieved at lower chemical concentrations and how stable the process would be with dirty, real samples. Both $\left(\mathrm{NH}_{4}\right)_{2} \mathrm{CO}_{3}$ and $\mathrm{Na}_{2} \mathrm{CO}_{3}$ are effective at $0.02 \mathrm{M}$. $\mathrm{Na}_{2} \mathrm{CO}_{3}$ can be seen to be effective at lower peroxide concentrations but the sodium adds to total solids in sample, which increases issues with fouling of the ICP-MS. 
Table 3.5. Extraction Performance ${ }^{(a)}$ from Different Swipe materials for Preferred Chemical Conditions

\begin{tabular}{l|ll|ll}
\hline \multirow{2}{*}{\multicolumn{1}{c|}{ Extractant }} & \multicolumn{2}{|c}{ \% Extraction from Muslin } & \multicolumn{2}{c}{ \% Extraction Muslin PTFE swipe } \\
\cline { 2 - 5 } & $\mathrm{U}_{3} \mathrm{O}_{8}$ & $\mathrm{UO}_{2} \mathrm{~F}_{2}$ & $\mathrm{U}_{3} \mathrm{O}_{8}$ & $\mathrm{UO}_{2} \mathrm{~F}_{2}$ \\
\hline $0.2 \mathrm{M}\left(\mathrm{NH}_{4}\right)_{2} \mathrm{CO}_{3}: 3 \mathrm{M} \mathrm{H}_{2} \mathrm{O}_{2}$ & $100( \pm 5)$ & $100( \pm 5)$ & $99( \pm 10)$ & $99( \pm 2)$ \\
$0.02 \mathrm{M}\left(\mathrm{NH}_{4}\right)_{2} \mathrm{CO}_{3}: 4 \mathrm{M} \mathrm{H}_{2} \mathrm{O}_{2}$ & $100( \pm 1)$ & $100( \pm 1)$ & $100( \pm 25)$ & $97( \pm 4)$ \\
$0.2 \mathrm{M} \mathrm{Na}_{2} \mathrm{CO}_{3}: 1 \mathrm{M} \mathrm{H}_{2} \mathrm{O}_{2}$ & $100( \pm 4)$ & $100( \pm 1)$ & $100( \pm 7)$ & $100( \pm 1)$ \\
$0.02 \mathrm{M} \mathrm{Na}_{2} \mathrm{CO}_{3}: 2 \mathrm{M} \mathrm{H}_{2} \mathrm{O}_{2}$ & $99( \pm 12)$ & $93( \pm 3)$ & $96( \pm 2)$ & $91( \pm 6)$ \\
\hline
\end{tabular}

(a) Average extraction percentage calculated from triplicate samples, and swipes contacted were treated within extraction solutions for 18 hours with continued shaking at $100 \mathrm{rpm}$.

\subsubsection{Summary}

Carbonate-peroxide chemistry is clearly effective extraction of hard (e.g., $\mathrm{U}_{3} \mathrm{O}_{8}$ ) and soft (e.g., $\mathrm{UO}_{2} \mathrm{~F}_{2}$ ) uranium species and can be used on existing (muslin) and other promising new sampling materials. In FY 2016 the research team showed a 100x reduction in solids and carbonated concentration from starting levels of $2 \mathrm{M}$ carbonate down to good extraction efficiency at $0.02 \mathrm{M}$. Another 10-100x reduction in solids/carbonated concentration is needed to enable direct assay of swipe leachate without affecting ICP-MS performance and additional work is needed determined if this can be achieved. If additional reductions in carbonate concentration are not possible, the carbonate-peroxide extraction is still a time-saving method and a modified procedure, suggested by ORNL can be used. The carbonate leachate is simply evaporated and the carbonate residue is ignited and reduced in a furnace. Similar to portions of the existing NWAL method, the residue can then simply be acidified, cleaned up with a microcolumn, and run on ICP-MS with good results.

Further study is needed to optimize and finalize a carbonate extraction protocol but several viable options have been shown exist that can produce a streamlined ES swipe extraction method that saves time and money.

\subsection{Evaluation and Validation of New Swipe/Sampling Materials}

Environmental sampling is a fundamental component of the IAEA's Safeguards regime. For decades, laboratory instrumentation, such as mass spectrometers, has undergone sustained development efforts to improve detection limits. However, the materials used for sampling have not changed since first implemented by the IAEA in the early 1990s. Better sampling materials and associated processing methods should benefit Safeguards efforts by reducing sample preparation cost and time, improving analytical performance, and increasing laboratory throughput. Previous NA-22 efforts reviewed and explored potential improvements that new sampling could provide for better analysis of environmental safeguard samples. Preferred sampling materials identified from the previous (NA-22) effort were selected for addition evaluation and validation.

Cotton is by far the most popular and universal sampling material. Its drape, tensile strength, and low cost are the most attractive aspects of this fabric. Cotton's relatively low levels of uranium are another reason that this material (specifically TexWipe TX304) is the current sampler of choice for IAEA environmental sampling use. A critical shortcoming with existing swipe material is the labor and time- 
intensive sample processing required (total digestion), increasing both the cost of laboratory analysis and the turnaround times needed to yield useful information and reducing laboratory throughput for ICP-MS, thermal ionization mass spectrometry (TIMS), and SIMS. Further, as cotton fibers are composed of natural cellulose, uranium levels cannot be expected to remain constant and have been found to vary significantly from batch to batch. To this end, a synthetic material, whose production and uranium levels can be tightly controlled, are likely the better choice as new and evolving analytical techniques become more sensitive to the background elements inherent to a sampling matrix. Further, while the cotton TexWipe TX304 (and any other cotton material) works, it does not enable fast, simple, or cost-effective analysis after collection. Consequently, alternative sampling materials with lower background and reduced sample processing time require merit evaluation and validation for improved analytical sample processing. Polymeric and carbon-based materials are particularly attractive because a wide range of commercial materials exist, the materials can be engineered into the desired form factor, and they polymers can be very low-background materials.

\subsubsection{Sampling Materials Selected for Evaluation of Bulk Uranium Extraction}

Based on previous work for NA-22 and additional assessments made in early FY 2016 five different sampling materials were selected for additional evaluation. The cotton-based TexWipe TX304 was used as a standard and baseline for comparative evaluation. Three promising synthetic materials were chosen; PTFE-coated cloth, surface-passivated fiberglass, and. carbon fiber materials. These three novel sampling materials are prototypes for evaluation (two were custom synthesized at PNNL) and further optimization of the material can be (and likely will need to be) made for a finalized sampling material. The materials are shown in Figure 3.2. Background on these materials is provided subsequently. Silver-coated nylon fabric was also included in portions of the study because it is a highly conductive material fabric that could potentially be used by SIMS; it was never intended to be used for bulk environmental sample analysis.

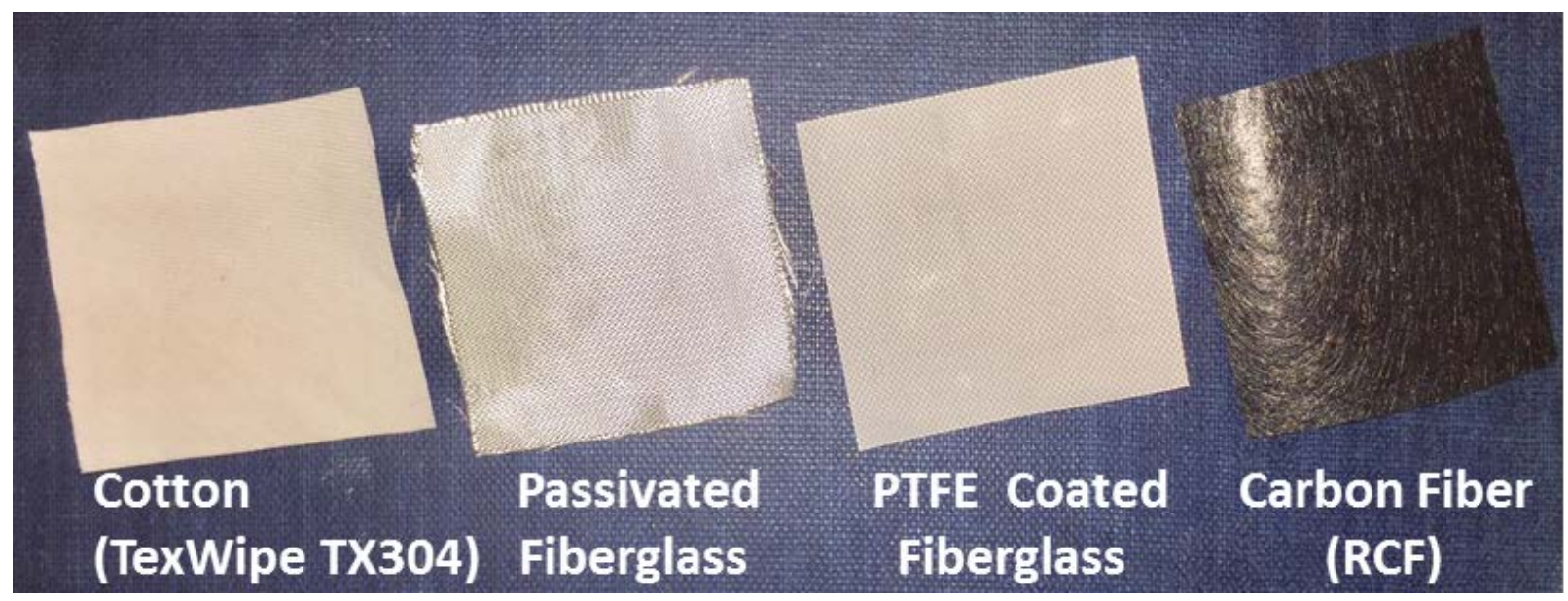

Figure 3.2. Samples of the Standard and Promising Environmental Sampling Materials. The cottonbased TexWipe TX304 is the standard material currently used. Three promising synthetic materials being evaluated are shown: surface/chemically passivated fiberglass, PTFE-coated fiberglass cloth, carbon fiber (RCF is shown but many other compositions available).

PTFE is a low-surface-energy material. The complete fluorination of its polymeric structure makes this material extremely inert and thermally stable. These qualities not only contribute to stabilization of 
collected analyte but also allow for a wide range of chemical treatments without running the risk of chemical degradation. PTFE-coated fabrics have some of the lowest surface energies known, providing excellent nonstick surface, enabling effective analyte release. These fabrics are used extensively for trace sampling and collection of explosives and narcotics. With desired properties such as low uranium background, excellent chemical stability, high tensile strength, flexibility, low surface energy, and a wide-range temperature stability, PTFE-coated fabrics were identified as an excellent candidate for development further investigation and evaluation. Additionally, when compared to muslin swipes, PTFE surfaces were found to have as good or better uranium particle collection and subsequently enabled a high removal/recovery of collected materials (especially with acidic leaching) for analysis. Commercial PTFE sampling materials are available. However previous (NA-22) efforts have found these materials to have higher uranium background (which could be cleaned and reduced for this application if there was a commercial market). For this effort, custom low-background PTFE-coated fiberglass materials were synthesized at PNNL with a proprietary method.

Passivation is a set of chemical processing techniques that render a material's surface chemically inactive. Passivation of sampling material surfaces makes them chemically inactive (similar to PTFE) and provides improved acid stability and analyte recovery. Silane chemistry is one route to surface passivation and has the advantage of being easily applicable to many materials. It is especially effective at passivating materials such as fiberglass cloth and steel mesh. Phenyl silane enables the assembly of a high-density surface layer of aromatic molecules that render a surface nonreactive to most chemical compounds. Further, aromatic phenyl silane layers are chemically and thermally very robust. Using phenyl silane to passivate cheaply sourced fiberglass offers a potential low-cost means of modifying fiberglass to produce effective sampling media. The research team found that high-density silane layers are successful in chemically passivating fiberglass sample cloth surfaces. For this effort custom surface passivated fiberglass material was synthesized by PNNL.

Carbon fibers, sometime called graphite fibers, are usually combined with other materials such as organics and resins to help with material processing, form composite material, improve the finish and enhance fabric properties. The carbon fibers offer desirable properties that make them a versatile swipe material candidate for uranium extraction with the bulk analysis method including metal-free, lowuranium background, thermal stability, high surface area, chemical resistance, and ease of activation or functionalization. The high surface area of some forms of carbon fiber can result in high chemical sorption capacity, while the thin cloth provides excellent adsorption kinetics and good surface contact. Moreover, carbon fiber can be blended or woven with other fabrics to enhance properties such as strength and abrasion resistance, and enable chemical functionalization. Because of these properties, carbon fibers have been developed for range of applications ranging from aerospace structures to sampling material. Although historically costly to produce, carbon fibers are now a relatively inexpensive, easy to source, and can be produced in a large variety of different weaves. The fibers are commercially available in woven and nonwoven pattern with different thicknesses. A range of carbon fiber materials is shown in Figure 3.3. Some of the carbon fiber fabrics provided promising properties for bulk analysis-sampling material and were therefore selected for further investigation. The carbon fabric selected for evaluation is a non-woven carbon fiber tissue, incorporating 100 percent carbon fiber bonded together in a random fiber matrix. Known as RCF carbon tissue, the dense fiber weave of carbon tissue material provides good conductivity (low resistivity), low roughness and low uranium background. The price per swipe is relatively low and previous efforts have shown it can be directly measured by SIMS. All of these 
properties made RCF a promising candidate and it was selected for further evaluation for both bulk assay as wells as direct SIMS analysis in FY 2016.

\subsubsection{Evaluation of Selected Bulk Environmental Sampling Materials}

Four types of uranium compounds; $\mathrm{UO}_{2}\left(\mathrm{NO}_{3}\right)_{2}, \mathrm{UO}_{2} \mathrm{~F}_{2}, \mathrm{U}_{3} \mathrm{O}_{8}$, and uranium ore concentrate (UOC) were chosen for deposition on selected swipe materials and the performance various extraction chemistries was explored. The method of uranium deposition (in microgram and nanogram levels commonly encountered with typical swipes) and extraction chemistries are explained in Section 3.1 and Appendix B.

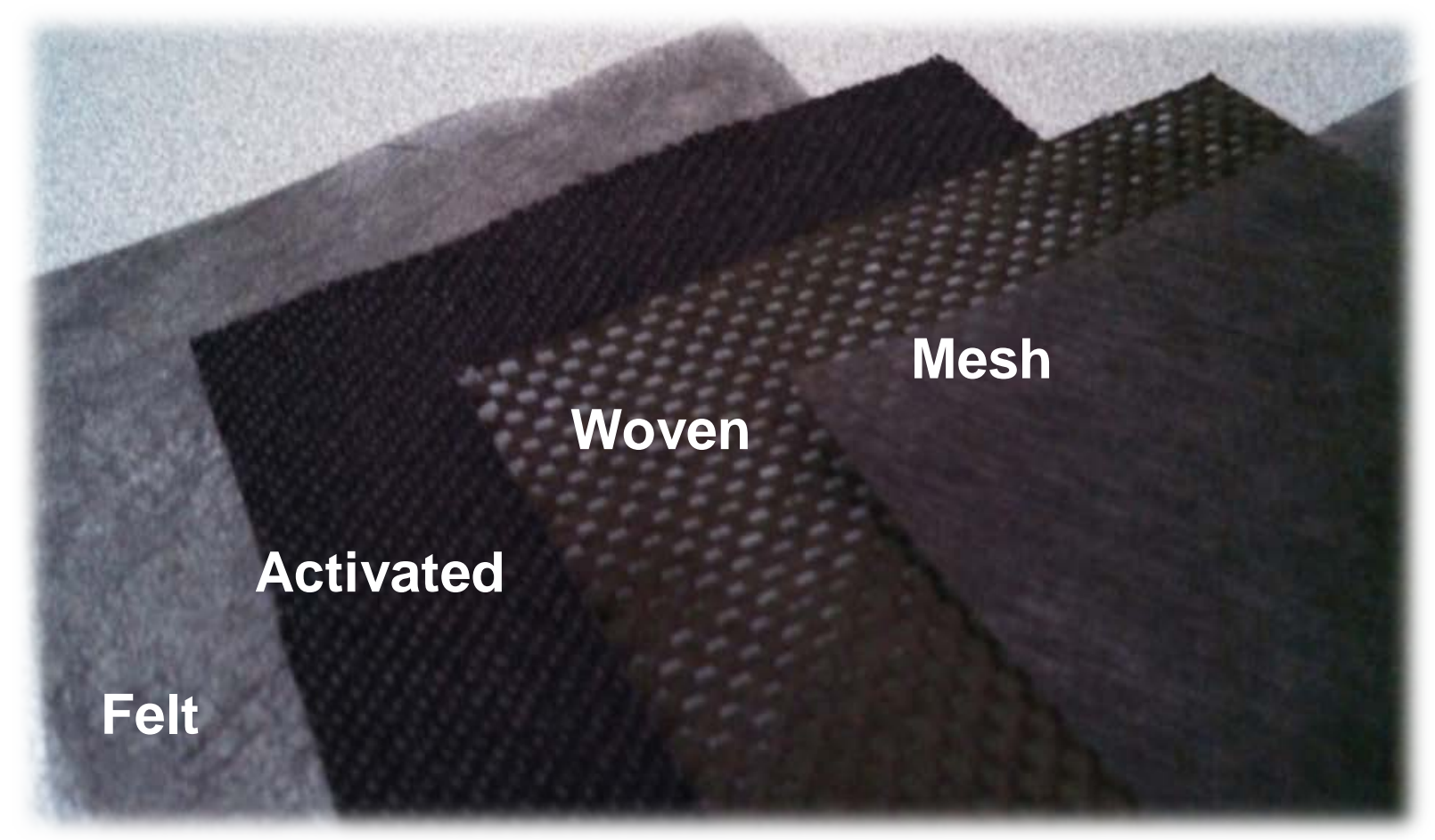

Figure 3.3. Different Carbon Fiber Materials. Fiber size, weave, and material density are all adjustable parameters. For bulk assay the materials can be cleaned to create very low background materials. For SIMS assay some of the materials are conductive, enabling direct assay of the sample.

$\mathrm{UO}_{2}\left(\mathrm{NO}_{3}\right)_{2}$ is a common reprocessing compound and represents a "worst-case scenario"/challenging analyte because of its chemical reactivity, especially toward silicate surfaces. In minutes $\mathrm{UO}_{2}\left(\mathrm{NO}_{3}\right)_{2}$ will react with typical silica surfaces and the extraction/recovery efficiency will fall off rapidly (silica can actually be used as a moderately effective uranium sorbent material). $\mathrm{UO}_{2} \mathrm{~F}_{2}$, is also a reactive compound and associated with enrichment efforts. $\mathrm{U}_{3} \mathrm{O}_{8}$ and uranium ore concentrate (UOC) are chemically stable compounds associated with nuclear fuel production. Table 3.6 summarizes recovery of these uranium compounds from selected sampling materials with extraction chemistries ranging from very mild (water) to aggressive acidic and caustic solutions. 
Background uranium extracted from swipe material is also shown in Table 3.6 for the various chemistries and can be seen to be elevated with the stronger extraction solutions. Ceramics of all types typically carry substantial quantities of uranium that could be extracted if the surface coating were completely or partially removed. Elevated background levels can be seen from the surface-passivated swipes and PTFE swipe with the more aggressive $6 \mathrm{M} \mathrm{HNO}_{3}$ and $2 \mathrm{M}\left(\mathrm{HN}_{4}\right)_{2} \mathrm{CO}_{3}-\mathrm{H}_{2} \mathrm{O}_{2}$ extraction solutions indicating some leaching from the fiberglass core fabric material. As expected the silver-coated nylon showed the highest uranium background content, which most likely came from silver metal coating leaching from the nylon fabric into extraction solutions.

Table 3.6. Background and Extraction Efficiency for Uranium Compounds from Selected Sampling Materials with Selected Extraction Chemistries

\begin{tabular}{|c|c|c|c|c|c|}
\hline \multirow{2}{*}{$\begin{array}{l}\text { Uranium } \\
\text { Compounds }\end{array}$} & \multirow{2}{*}{$\begin{array}{l}\text { Swipe } \\
\text { Material }\end{array}$} & \multicolumn{4}{|c|}{ \% Extraction Efficiency } \\
\hline & & Water & $0.01 \mathrm{M} \mathrm{HNO}_{3}$ & $6 \mathrm{M} \mathrm{HNO}_{3}$ & $\begin{array}{c}2 \mathrm{M}\left(\mathrm{HN}_{4}\right)_{2} \mathrm{CO}_{3}: \\
2 \mathrm{M} \mathrm{H}_{2} \mathrm{O}_{2}\end{array}$ \\
\hline \multirow[t]{5}{*}{$\mathrm{UO}_{2}\left(\mathrm{NO}_{3}\right)_{2}$} & TexWipe TX304 & $32(+/-3)$ & $84(+/-2)$ & $100(+/-1)$ & $100(+/-1)$ \\
\hline & PTFE & $40(+/-2)$ & $100(+/-1)$ & $100(+/-1)$ & $97(+/-3)$ \\
\hline & Passivated & $94(+/-3)$ & $100(+/-1)$ & $100(+/-5)$ & $88(+/-7)$ \\
\hline & Carbon Fiber & $11(+/-7)$ & $47(+/-3)$ & $100(+/-2)$ & $86(+/-7)$ \\
\hline & Ag Coated Nylon & $27(+/-2)$ & $50(+/-10)$ & $100(+/-3)$ & NA \\
\hline \multirow[t]{5}{*}{$\mathrm{UO}_{2} \mathrm{~F}_{2}$} & TexWipe TX304 & $46(+/-4)$ & $86(+/-5)$ & $100(+/-1)$ & $100(+/-1)$ \\
\hline & PTFE & $24(+/-4)$ & $97(+/-5)$ & $100(+/-1)$ & $99(+/-1)$ \\
\hline & Passivated & $59(+/-10)$ & $91(+/-10)$ & $100(+/-10)$ & $82(+/-5)$ \\
\hline & Carbon Fiber & $2(+/-1)$ & $51(+/-10)$ & $97(+/-5)$ & $70(+/-6)$ \\
\hline & Ag Coated Nylon & $13(+/-3)$ & $41(+/-4)$ & $96(+/-4)$ & $76(+/-10)$ \\
\hline \multirow[t]{5}{*}{ UOC } & TexWipe TX304 & $41(+/-4)$ & $64(+/-3)$ & $70(+/-1)$ & $100(+/-5)$ \\
\hline & PTFE & $38(+/-2)$ & $65(+/-9)$ & $73(+/-10)$ & $100(+/-4)$ \\
\hline & Passivated & $48(+/-10)$ & $66(+/-3)$ & $98(+/-2)$ & NA \\
\hline & Carbon Fiber & $13(+/-5)$ & $42(+/-5)$ & $98(+/-7)$ & $85(+/-10)$ \\
\hline & Ag Coated Nylon & $4(+/-2)$ & $43(+/-1)$ & $100(+/-1)$ & $96(+/-10)$ \\
\hline \multirow[t]{5}{*}{$\mathrm{U}_{3} \mathrm{O}_{8}$} & TexWipe TX304 & $3(+/-1)$ & $47(+/-5)$ & $58(+/-1)$ & $98(+/-4)$ \\
\hline & PTFE & $3(+/-1)$ & $49(+/-2)$ & $70(+/-11)$ & $98(+/-1)$ \\
\hline & Passivated & $2(+/-1)$ & $32(+/-10)$ & $99(+/-8)$ & NA \\
\hline & Carbon Fiber & $4(+/-1)$ & $10(+/-1)$ & $91(+/-1)$ & $100(+/-10)$ \\
\hline & Ag Coated Nylon & $11(+/-1)$ & $15(+/-1)$ & $100(+/-5)$ & $74(+/-4)$ \\
\hline \multirow[t]{5}{*}{ Background } & TexWipe TX304 & $<1 \mathrm{ng} / \mathrm{g}$ & $<1 \mathrm{ng} / \mathrm{g}$ & $<1 \mathrm{ng} / \mathrm{g}$ & $<1 \mathrm{ng} / \mathrm{g}$ \\
\hline & PTFE & $<1 \mathrm{ng} / \mathrm{g}$ & $<1 \mathrm{ng} / \mathrm{g}$ & $1.8 \mathrm{ng} / \mathrm{g}$ & $1.5 \mathrm{ng} / \mathrm{g}$ \\
\hline & Passivated & $<1 \mathrm{ng} / \mathrm{g}$ & $<1 \mathrm{ng} / \mathrm{g}$ & $3.3 \mathrm{ng} / \mathrm{g}$ & 3.9 ng/g \\
\hline & Carbon Fiber & $<1 \mathrm{ng} / \mathrm{g}$ & $<1 \mathrm{ng} / \mathrm{g}$ & N/A & $<1 \mathrm{ng} / \mathrm{g}$ \\
\hline & Ag Coated Nylon & $<1 \mathrm{ng} / \mathrm{g}$ & $13.6 \mathrm{ng} / \mathrm{g}$ & 485 ng/g & $510 \mathrm{ng} / \mathrm{g}$ \\
\hline
\end{tabular}

Samples were prepared and analyzed in triplicate. Swipes were treated within extraction solutions for 18 hours. Carbon fiber material used was RCF carbon tissue. N/A = Some data are not available. 
As expected Table 3.6 shows the more aggressive extraction conditions (6 $\mathrm{M} \mathrm{HNO}_{3}$ and the peroxidecarbonate solution) to be more effective with high extraction efficiencies from all most materials for most uranium compounds. Milder extraction conditions $\left(0.01 \mathrm{HNO}_{3}\right.$ and water) can be surprisingly effective at recovering uranium particles from chemically passive and inert materials (passivated and PTFE swipes). Extraction efficiencies uranium oxide and minerals ( $\mathrm{UOC}$ and $\mathrm{U}_{3} \mathrm{O}_{3}$ ) are generally lower than the more reactive uranium species $\left(\mathrm{UO}_{2} \mathrm{~F}_{2}\right.$ and $\left.\mathrm{UO}_{2}\left(\mathrm{NO}_{3}\right)_{2}\right)$. Extraction efficiencies with mild solutions from the chemical nonreactive materials (passivated and PTFE swipes) are shown to be very effective for the reactive uranium species $\left(\mathrm{UO}_{2} \mathrm{~F}_{2}\right.$ and $\left.\mathrm{UO}_{2}\left(\mathrm{NO}_{3}\right)_{2}\right)$ associated with nuclear processing activities. Additional efforts should explore the swipe materials efficacy with lower carbonate concentrations to enable lower salt and solids content in the leachate.

Both nonconductive materials explored (passivated and PTFE swipes) showed performance as good or better than the standard cotton-based swipe for all extraction conditions. PTFE, and especially passivated swipes were particularly effective with mild extraction solutions for $\mathrm{UO}_{2}\left(\mathrm{NO}_{3}\right)_{2}$ and $\mathrm{UO}_{2} \mathrm{~F}_{2}$, outperforming all other materials evaluated (Table 3.6). With passivated and PTFE swipes the milder extraction conditions ( $0.01 \mathrm{M} \mathrm{HNO}_{3}$ and water) are surprisingly effective at recovery from the sampling material of the more reactive uranium particles that are associated with uranium processing activities. One issue with the passivated swipes is the increase in background with the $2 \mathrm{M}\left(\mathrm{HN}_{4}\right)_{2} \mathrm{CO}_{3}-\mathrm{H}_{2} \mathrm{O}_{2}$. This is likely because of the basic extraction conditions removing some of the phenyl silane surface passivation layer and extracting some uranium for the core fiberglass material.

For conductive swipe material, carbon fiber and silver-coated nylon showed excellent extraction performance for the full range of uranium compounds with strong acid solutions, generally good performances in carbonate-peroxide solution, and poor performance in mild condition. The results indicate that carbon fiber can be used for uranium collection and subsequent examination with bulk analysis technique with aggressive extraction solutions. Moreover, as discussed in greater detail in Section 4.2, carbon fiber is electrically conductive, which enables direct SIMS analysis of uranium particles on the swipe. This makes the carbon fiber a very attractive material for environmental sampling because it can be used for both particle and bulk uranium analysis. However, the specific carbon fiber fabric(s) which would be ideal for this use have not yet been identified. Carbon fiber materials with more physical durability (to avoid fraying and fragmenting during sampling and processing) as well as improved flatness (for SIMS analysis) needs to be identified. The silver-coated nylon was not suitable for uranium bulk analysis because of its high background uranium content. This material needs further investigation to determine whether it is suitable for uranium examination with SIMS analysis.

\subsubsection{Summary}

In summary, many of the alternative sampling materials showed low backgrounds and better extraction performance than the standard TexWipe TX304. The alternative materials also enable simplified extraction processes (with reduced digested and sample treatment steps) and low backgrounds. Some materials (passivated and PTFE swipes) enable enhanced extraction efficiencies under mild conditions for the reactive uranium species $\left(\mathrm{UO}_{2} \mathrm{~F}_{2}\right.$ and $\left.\mathrm{UO}_{2}\left(\mathrm{NO}_{3}\right)_{2}\right)$ associated with nuclear processing activities. Some materials enable both bulk extraction as well as SIMS analysis (select carbon fiber materials). Additional work is needed to iteratively optimize these alternative sampling materials to provide an improved bulk uranium extraction process for nuclear safeguard efforts. 


\subsection{Task 2. Optimize Materials and Methods for Direct SIMS Environmental Sample Analysis}

SIMS provides isotopic identification and ratios for specific particles collected from sampling materials. SIMS has long been used for the analysis of uranium isotopes for individual particles. A stateof-the-art system is online at the IAEA in Vienna, and many other NWAL facilities have a SIMS system in place or on order. In FY 2016, a new NWAL-compatible state-of the-art SIMS system came online at PNNL. The current method for analyzing swipe particulates is time- and labor-intensive. Further, the particle transfer process (from muslin swipe to SIMS sample mount) presents an inefficient analytical bottleneck that is manually intensive, slows sample throughput, and increases background levels.

This task developed and demonstrated sampling materials and analysis methods that that provide faster, simpler, and more cost-effective isotopic assay of particles with SIMS. The research team moved beyond previous proof-of-principal efforts (supported by NA-22) and maturing SIMS technology by integrating and optimizing the material and methods. The existing technique and new approach are shown in the conceptual graphic in Figure 4.1.

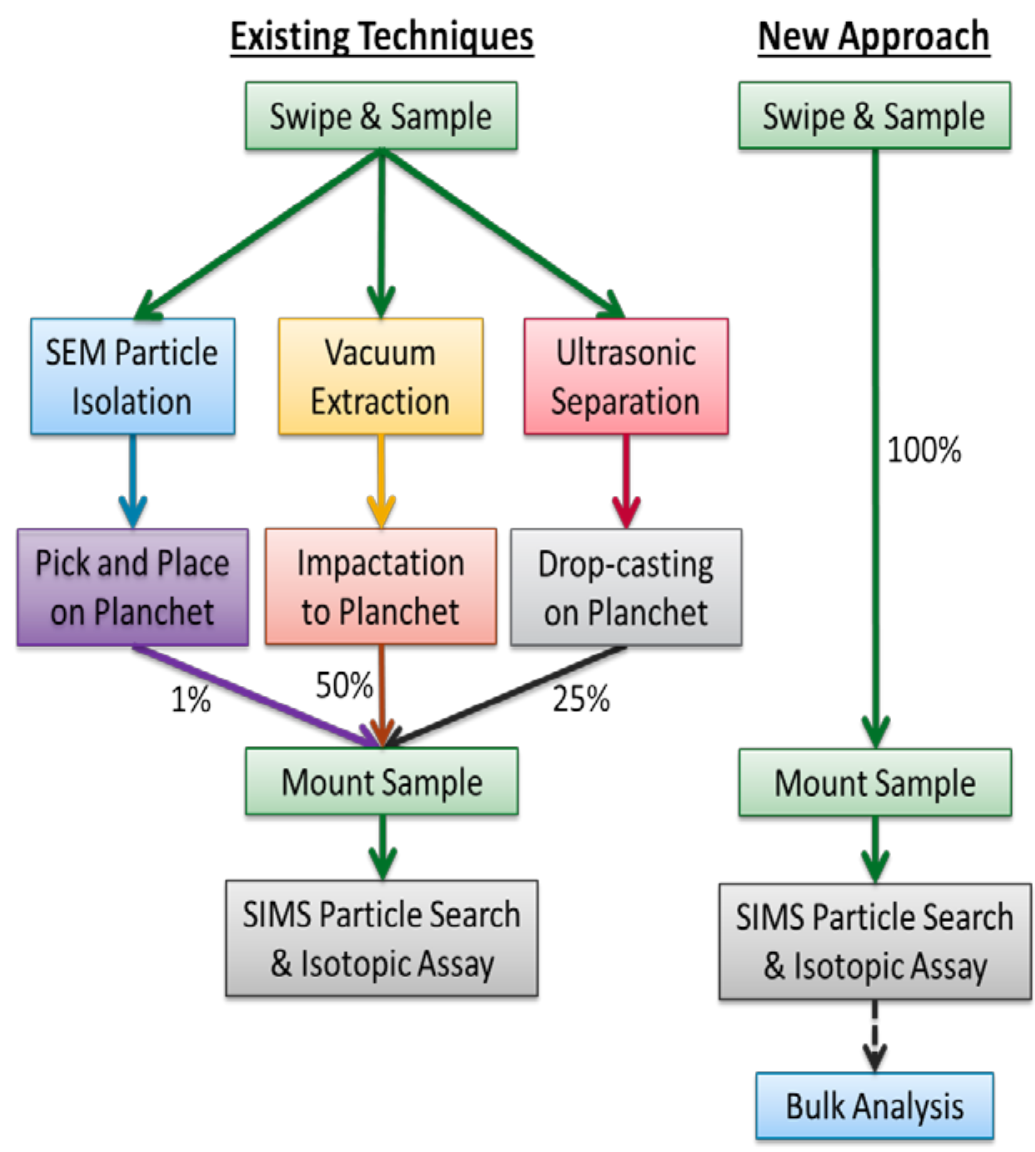

Figure 4.1. Comparison of the Existing Technique and the New Approach for Preparation and Analysis with SIMS for Particle Assay. The methods and materials under development provide simpler, faster, and more cost effective methods for measurement of safeguard environmental samples. 
The focus of Task 2 was optimizing materials and methods to enable direct SIMS assay of environmental swipe samples. The task was divided into 2 primary efforts areas with specific focus areas of interest that were delineated below. Not all areas of interest could be explored in FY 2016 efforts.

1. SIMS Methods Development

- Sample mounting

- Sample automation

- $\quad$ Rapid searching methods

- Sample analysis

2. Improved Swipe/Sampling Material for SIMS that Enable Direct Assay

- $\quad$ SIMS-compatible materials

- High surface conductivity

- Effective sampling fabric texture

- Flatter is better for SIMS assay

- Commercially available

- Materials compatible with bulk assay (optional but enables one sampling material to provide all ES sampling functions)

- Inert in acid or base digestion processes

○ Nonreactive with enrichment samples

$\circ \quad$ Ultralow and stable background

FY 2016 results for these efforts are detailed subsequently in Sections 4.1-4.3 and summarized below.

- A SIMS sample holder that retrofits into existing equipment and provides simple effective rapid mounting of swipe sample for direct assay while enabling automation and laboratory integration was designed.

- Preferred conductive sampling materials with better performance characteristics were identified.

- Low level environmental samples were successfully run on the new PNNL Cameca 1280 SIMS.

- Excellent agreement between isotopic ratios for certified materials and direct SIMS assay of very low levels of low enriched uranium (LEU) and highly enriched uranium (HEU) uranium oxyofluoride species (UOF) particles on carbon fiber sampling material. Sample activities range from 1 to 500 counts per minute (cpm). The uranium mass on a sample was dependent upon the specific isotope ratio, but was frequently in the subnanogram range.

- The research team found the presence of the UF molecular ions, as measured by SIMS, provided chemical information about the particle that was separate from the uranium isotopics and strongly suggested that those particles originated from an $\mathrm{UF}_{6}$ enrichment activity.

- The research team submitted one manuscript, and another is being drafted.

- The research team submitted a final report containing FY 2016 results for efforts on Task 2. 


\subsection{SIMS Environmental Sample Methods Development}

SIMS is a powerful analytical tool for measuring isotopic distributions of uranium-bearing particles and can provide key information for a range of safeguards relevant samples. SIMS can identify and quantify isotopic ratios of individual particles with a high discrimination against background because the particles are assayed individually. Method improvements that enable direct and more automated SIMS analysis will eliminate laborious sample handling and would allow this powerful method to be more widely used by the safeguards community. The research team explored methods to improve SIMS, including sample mounting and rapid search and assay techniques.

Searching for individual enriched particles on swipe sample is a key challenge for SIMS throughput. Finding individual particle particles showing enrichment activity on environmental samples is modern analog of the old statement concerning "finding a needle in a haystack." Improved search routines with the SIMS systems have been explored by PNNL (Willingham et. al. 2016) and are a way to automate and accelerate the process. The fabric of the sampling material is not a perfect planar surface of typical (prepared) SIMS samples. Particles on sampling cloth can move in and out of the SIMS field of view. Consequently the flattest possible sampling cloth is needed (along with effective flat mounting) as well as automated methods to adjust for changes in the plane of focus for the local field of view (future work). Using orthogonal complementary screening methods to identify areas of interest on the sample is another way to focus the SIMS assay on the regions of interest. Methods that merit consideration as SIMS screening techniques include Digital Autoradiography Imaging (a current NA-241 sponsored project at PNNL, being conducted by Brain Miller PI), XRF and possibly optical methods (i.e., Raman and fluorescence). Exploring rapid screening methods (both with the SIMS system and with coordinated complementary techniques) will be a productive focus for future methods development efforts.

A key component to SIMS work is sample preparation. Preparing quantitative samples with the desired isotopic ratio, particle size ( 1-100 microns), particle chemistry (e.g., UOF) and correct particle ratio (a small fraction of enriched particles in a sea of "normal” particles) is nontrivial. This effort is described in Section 6.

\subsubsection{Improved Sample Mounting for Direct Assay of Swipe Sampling Cloth}

Methods to rapidly prepare/mount the swipe sampling materials for SIMS assay are advantageous, particularly if they enable laboratory automation and information systems. An improved SIMS sample mount would:

- Retrofit into existing SIMS sample mounts (for planchets)

- Hold fabric samples very flat (to help keep sample in focal plane of instrument)

- Provide needed conductivity

- Enable simple snap fit assembly

- Enable bar coding/automated sample tracking

- Enable focusing on regions of interest

- Enable future automation of SIMS. 
Figure 4.2 is a schematic for an improved sample mount that should enable, faster, simpler automated direct SIMS assay of (conductive) environmental samples.

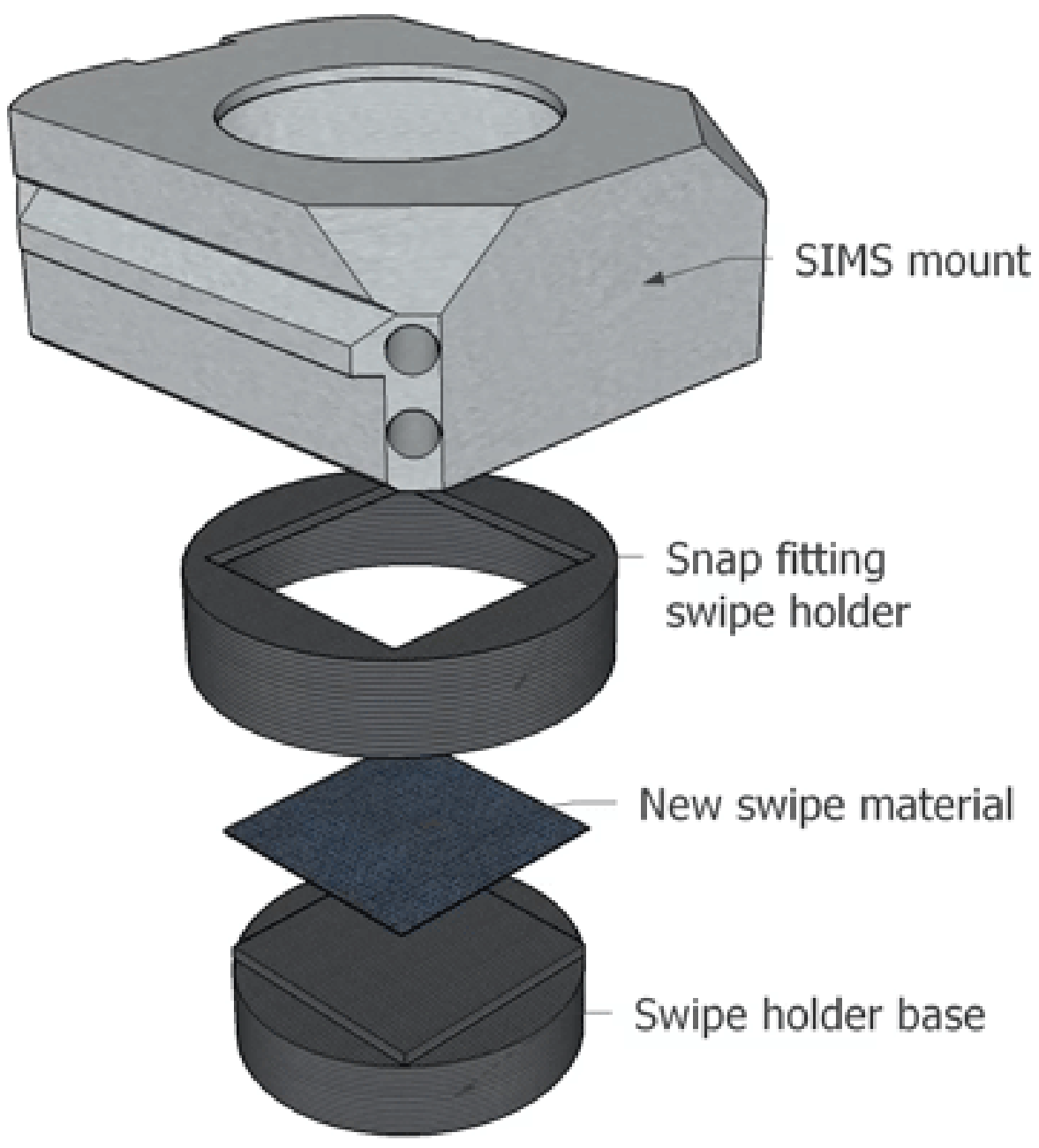

Figure 4.2. A Schematic for Sample Mount that Enables Direct Assay of (conductive) Environmental Swipe Samples with SIMS. The mount is designed to enable faster, simpler automated SIMS assay of environmental samples.

The components would slide directly into a standard SIMS mount (upper component of in Figure 4.2) and function in a standard CAMECA 1280 SIMS without modification. The "snap fitting" holder slides over the "swipe holder base" and locks the swipe sample material in place in a fashion similar to a common plastic storage (e.g., Tupperware ) lid snapping closed on its container. The swipe holder base has a slightly elevated stage to hold the fabric samples very flat. Flat is important since SIMS is a particle imaging technique, and maintaining the sample surface (with the particles of potential interest) in the focus is essential for data collection. The simple snap fit assembly makes mounting of the sample easy and provides some tension on the sample fabric to help keep it flat. Tolerances must be adjusted between the holder base and upper snap fitting, depending upon the swipe material thickness. The mounted sample (the fabric secured between the base holder and snap fit top) can have bar code attached (e.g., on the bottom) to enable sample tracking and coordination with laboratory information systems. With the fabric 
sample securely mounted (between the snap top and base) $x-y$ orientation is maintained and the mounted sample can be transferred between screening instruments and the SIMS without loss of orientation on the regions of interest. Once mounted (between the snap top and base) and barcoded, the sample could be easily moved and tracked between instruments and laboratories in a convenient form.

All components in sample mounting system must be conductive to enable SIMS assay and avoid issues with charging of the sample during analysis. Traditionally SIMS mounting components are expensive and precision machined from expensive stainless steel. The research team found that the lower components (the swipe base mount and the snap fit top) can be made from low-cost conductive polymers and or even be produced with a 3-D printer. Surprisingly these polymer components appear to be vacuum-stable and sufficiently conductive to enable SIMS. The polymers used in the 3D printed mount included acrylonitrile butadiene styrene (ABS, a common thermoplastic polymer) for nonconductive components. Conductive poly(lactic acid) or PLA. PLA is made conductive with the addition of graphene/graphite carbon to the polymer). Future efforts might also show an entire SIMS mount could be made inexpensively from 3D-printed conductive polymers.

\subsection{Direct Swipe Sample Analysis with SIMS}

An evaluation challenging trace level enrichment samples on conductive carbon fiber sampling material was done with SIMS to show the viability of the proposed approach. The Zorflex FM50Kactivated carbon cloth was used as the sampling material because it is one of the most suitable materials identified (to date) to enable direct SIMS assay of surface samples. After the surface sampling, the activated carbon cloth was mounted directly in a standard SIMS sample holder and introduced into the SIMS instrument for measurement without any additional processing steps (according to the process shown in Figure 4.1). Certified uranium standard materials with known isotopic ratios were used to generate low-level UF6 samples, which rapidly converted to UOF via hydrolysis and time. Section 6 provides details on the sample prep methods. Table 4.1 shows SIM data from an HEU and an LEU sample of $\mathrm{UF}_{6} / \mathrm{UOF}$ samples (prepared according to the process described in Section 6). Excellent agreement can be seen in Table 4.1 between in isotopic ratios for certified materials and direct SIMS assay of swipe materials with low levels of both the LEU and HEU UOF particles on carbon fiber sampling material.

Table 4.1. Comparative SIMS Results for Enriched $\mathrm{UF}_{6}$ Samples $^{(\mathrm{a})}$ on Carbon Fiber Sampling Material

\begin{tabular}{|c|c|c|c|c|}
\hline & \multicolumn{2}{|c|}{ Direct SIMS Assay on Carbon Fiber Cloth } & \multicolumn{2}{|c|}{ Certified Standard Values } \\
\hline & $\%{ }^{235} \mathrm{U}$ & $\%{ }^{238} \mathrm{U}$ & $\%{ }^{235} \mathrm{U}$ & $\%{ }^{238} \mathrm{U}$ \\
\hline LEU & 2.7 & 97.3 & 2.6 & 97.4 \\
\hline $\mathrm{HEU}$ & 94.3 & 5.7 & 93.1 & 5.9 \\
\hline
\end{tabular}

(a). Samples from trace $\mathrm{UF}_{6}$ vapor deposition (as described in Section 6) on the carbon fiber material Zorflex 50.

The SIMS could accurately detect $\mathrm{UF}_{6} / \mathrm{UOF}$ particles directly deposited upon the sample material. The SIMS could also accurately detect $\mathrm{UF}_{6} / \mathrm{UOF}$ that had been deposited on dust (mineral) particles and subsequently collected from other surfaces with the carbon fiber sampling material. Accurate particle and isotope ratios were determined directly on (carbon fiber) swipe samples with activities ranging from 1 to $500 \mathrm{cpm}$. The uranium mass on the sample is dependent upon specific the isotope ratio but it is frequently in the subnanogram range. Samples were successfully run on the new Cameca 1280 SIMS at PNNL. 


\subsubsection{Validation of Enrichment Activities with a Chemical Signature from SIMS Assay}

The isotopic ratio of the uranium components of a sample are typically considered the key information obtained in SIMS particle analysis (as well as bulk analysis) of an environmental sample. SIMS assay may be able to provide additional key orthogonal chemical information for the environmental samples, which enable direct correlation with enrichment efforts. The typical means for uranium isotopic enrichment uses centrifugal technology with $\mathrm{UF}_{6}$ gas. Uranium compounds originating from this process are uranium oxyofluoride species. Consequently association of fluorine $(F)$ with the uranium in a particle is a very strong indication that the particle originated as a result of enrichment activities.

In addition to measuring uranium isotope ratios in particles, SIMS can also measure molecular ions emanating from the sample, such as uranium oxide and uranium fluoride compounds. Samples (particles) with deposits of $\mathrm{UF}_{6}$ (converted to UOF via hydrolysis and time) persistently show UF ion fragments. Figure 4.3 shows SIMS data for a single field of view (FOV) on a carbon fiber cloth sample that had been used to collect dust from a surface that had low levels of $\mathrm{UF}_{6}$ particles deposited upon it (particles that undoubtedly converted to UOF prior to SIMS analysis). It can be seen that the SIMS signals within the same FOV for both ${ }^{235} \mathrm{U}$ and ${ }^{238} \mathrm{U}$, correlate directly with the corresponding UF signals- this is not observed for particles originating from non-UF 6 processes. The presence of the UF molecular ions, as measured by SIMS, provides chemical information about the particle that is separate from the uranium isotopics and strongly suggests that those particles originated from an $\mathrm{UF}_{6}$ enrichment activity.


Figure 4.3. SIMS Images for a Single Field of View on a Carbon Fiber Cloth Sample that had been Used to Collect Dust from a surface that had Low Levels of $\mathrm{UF}_{6}$ Particles. The presence of the UF SIMS signal, particularly the ${ }^{238} \mathrm{U}^{19} \mathrm{~F}$ (257 atomic mass unit [amu]) is a strong, clear and distinctive indicator that the particle(s) under examination have fluorine and likely originate from enrichment activity.

It should be noted that the atomic mass summation for ${ }^{235} \mathrm{U}^{19} \mathrm{~F}(254 \mathrm{amu})$ is the same mass as ${ }^{238} \mathrm{U}^{16} \mathrm{O}$ and is consequently unresolvable in the configuration by which the signals were obtained. However, ${ }^{238} \mathrm{U}^{19} \mathrm{~F}$ is a strong, clear and distinctive indicator that the particle(s) under examination are likely UOF species that originated from an enrichment process. 


\subsection{Conductive Fabric Sampling/Materials to Enable Direct SIMS Assay}

The current methods for analyzing swipe particulates (graphically shown in Figure 4.1) uses either sonication of a sampler in a Freon solvent or vacuum impaction to extract particulates from the surface of a swipe. The narrow stream of material sucked into the system is directed at a planchet of either a glassy carbon or pyrolitic graphite. In most cases, the weak interactions between the surface and particle stream are sufficient for adhesions; however, a vacuum grease coating is used (on the sample planchet/stage) in instances of low sample concentration. The current particle transfer process is a somewhat inefficient (25 to 50 percent transfer to planchet) analytical bottleneck that is manually intensive, slows sample throughput, and increases background levels. Direct assay of the sampling/swipe material would be significantly less labor- and time-intensive. A sampling material that enabled direct SIMS assay would allow SIMS to become more widely used and enable high throughput (isotopic) particle identification and assay in IAEA and NWAL laboratories.

Conductive sampling materials would enable direct SIMS assay, thereby avoiding time-consuming and labor-intensive sample processing. Previous work for NA-22 had shown the some carbon fiber cloth materials were very promising material for enable direct SIMS assay. The materials were conductive, effective for particle pick-up and had very low uranium backgrounds (see Table 3.6 for representative comparative values). In particular, the two carbon fiber materials, RCF Carbon Tissue and Zorflex50 Activated Carbon Cloth, showed very promising performance. However, further performance improvements in sampling material strength/integrity, conductive and morphology would be desirable

In FY 2016 the research team explored conductive swipe materials further to determine which materials could provide effective collection and enable direct SIMS measurement without the laborintensive sample preparation steps. The objective was to find materials that would speed up assay and increase laboratory throughput. Ideally the cloth would also be compatible with bulk ES processing (Task 1, Section 3). The desired properties should include characteristics such as good particle pick-up efficiency, high conductivity, high material strength and fabric cohesion, low surface roughness and low uranium background. In selecting materials to evaluate, electrical conductivity and surface roughness were weighted more heavily in the list of material properties. Conductive (low electrical resistivity) materials enable direct SIMS analysis and avoid charging of the sample surface from the ion beam. Surface morphology is key because it affects the particles in the field of view for SIMS assay. It is generally accepted that this depth of field is in the 1-10 micron range, but the exact values are generally dependent upon the sample and instrument configuration. Consequently, a flat surface topography is desired so that a larger percentage of the collected particles is within the SIMS focus depth for sample analysis. Sampling materials with relatively low surface roughness are preferred. Table 4.2 shows the electrical resistivity and surface roughness (macro and microscales) of materials selected for FY 2016 evaluation. Measurement methods are provided in Appendix C.

Low uranium background is still important (particularly for compatibility with bulk assay) but of less concern for direct SIMS assay (compared to electrical resistivity and surface roughness) because the field of view is limited (thereby limiting the mass of background uranium). Further, residual background uranium can be cleaned from carbon fiber materials with acidic or basic leaching processes (as shown in Table 3.6). 
Table 4.2. Properties of Selected Sampling and Reference Materials

\begin{tabular}{|c|c|c|c|}
\hline Sampling Material & $\begin{array}{l}\text { Electrical } \\
\text { Resistivity } \\
(\Omega \text {-cm) }\end{array}$ & $\begin{array}{l}\text { Macro- } \\
\text { Roughness }^{(\mathrm{a})} \\
(\mu \mathrm{m})\end{array}$ & $\begin{array}{c}\text { Micro-Roughness }^{(\mathrm{b})} \\
(\mu \mathrm{m})\end{array}$ \\
\hline \multicolumn{4}{|l|}{ Carbon Based Materials } \\
\hline CNT fabric & $1.9 \times 10^{-1}$ & 11 & 5 \\
\hline Carbon twill weave $19.8 \mathrm{oz}$ & $1.0 \times 10^{0}$ & 15 & 11 \\
\hline Carbon fabric $3.5 \mathrm{oz}$ & $1.1 \times 10^{0}$ & 26. & 12 \\
\hline Carbon Tissue (RCF) & $1.5 \times 10^{0}$ & 53 & 47 \\
\hline Graphite fabric $5.7 \mathrm{oz}$ & $3.5 \times 10^{0}$ & 17 & 8 \\
\hline Graphite Veil, $0.22 \mathrm{oz}$ & $3.7 \times 10^{0}$ & 60 & 66 \\
\hline Graphite fabric, $10.9 \mathrm{oz}$ & $4.1 \times 10^{0}$ & 15 & 15 \\
\hline Carbon twill weave $10.9 \mathrm{oz}$ & $5.9 \times 10^{0}$ & 22 & 5 \\
\hline $\begin{array}{l}\text { Activated carbon fiber cloth } \\
\text { (Zorflex-double weave) }\end{array}$ & $13.4 \times 10^{0}$ & 46 & 1 \\
\hline Graphite twill weave $5.7 \mathrm{oz}$ & $15.8 \times 10^{0}$ & 22 & 22 \\
\hline Unidirectional carbon $9 \mathrm{oz}$ & $20.7 \times 10^{0}$ & 8 & 2 \\
\hline $\begin{array}{l}\text { Activated carbon fiber cloth } \\
\text { (Zorflex-single weave) }\end{array}$ & $23.8 \times 10^{0}$ & 51 & 52 \\
\hline Activated Carbon Cloth (Zorflex FM50K) & $34.1 \times 10^{0}$ & 34 & 16 \\
\hline Activated carbon cloth (Zorflex-VB ${ }^{+}$) & $46.4 \times 10^{0}$ & 45 & 21 \\
\hline \multicolumn{4}{|l|}{ Metalized Composite Nylon Materials ${ }^{(\mathrm{c})}$} \\
\hline Ni Ag coated Non-Woven Point Bond & $7.7 \times 10^{-2}$ & 49 & 3 \\
\hline Ni Ag coated Nylon Ripstop & $4.8 \times 10^{-2}$ & 15 & 11 \\
\hline Ni Ag coated Nylon Taffeta & $6.2 \times 10^{-2}$ & 11 & 7 \\
\hline Ag coated Nylon Ripstop & $6.6 \times 10^{-2}$ & 15 & 10 \\
\hline Ni Ag coated Non-Woven & $1.4 \times 10^{-1}$ & 48 & 27 \\
\hline Ag coated Nylon Tricot Knit & $1.9 \times 10^{-1}$ & 57 & 36 \\
\hline Ag coated Tricot Knit & $2.2 \times 10^{-1}$ & 69 & 31 \\
\hline Ag coated Nylon Hook & $9.2 \times 10^{-1}$ & 39 & 10 \\
\hline Ag coated Nylon Jersey knit & $1.1 \times 10^{0}$ & $\mathrm{NE}$ & $\mathrm{NE}$ \\
\hline Ag coated Continuous Nylon Loop & $1.1 \times 10^{0}$ & $\mathrm{NE}$ & $\mathrm{NE}$ \\
\hline Ag coated Nylon Knit (48x21) & $1.5 \times 10^{0}$ & 34 & 35 \\
\hline Ag coated Nylon Loop & $2.8 \times 10^{0}$ & NE & $\mathrm{NE}$ \\
\hline \multicolumn{4}{|l|}{ Comparative Materials } \\
\hline Cu Mesh & $9.1 \times 10^{-2}$ & NA & NA \\
\hline TexWipe 304 & $5.9 \times 10^{7}$ & 31 & 13 \\
\hline \multicolumn{4}{|c|}{$\begin{array}{l}\text { (b) Micro roughness was estimated from area of } 0.01188 \mathrm{~mm}^{2} \text {. } \\
\text { (c) All Metalized Composite Materials on nylon and supplied by Swift Textile Metalizing. } \\
\text { NA = None available; NE = None estimable because of the fuzzy/uneven surface of the fabric } \\
\text { Measurement methods can be found in Appendix C. }\end{array}$} \\
\hline
\end{tabular}


Table 4.2 shows the electrical resistivity and surface roughness (macro and microscales) of the materials evaluated for electrical conductivity and surface roughness (uranium background information can be found in Table 3.6). A range of materials different conductive materials were explored including carbon-based materials and metalized composite materials. Values for a completely conductive metal mesh and the standard IAEA sampling material (cotton and nonconductive) are provided at the bottom of Table 4.2 for comparison. RCF (carbon tissue) and Zorflex FM 50K (activated carbon cloth), the promising novel candidates materials previously identified on the NA-22 program, were also included for comparative evaluation. Carbon tissue is a random non-woven mixture of carbon fibers. Activated carbon cloth is a fabric of activated carbon fibers with high porosity and a larger surface area. Both types of materials have a propensity to fray, flake, and shed material making them less than ideal. Ideally, the research team would like to find a flat (low surface roughness), conductive (low resistivity) material that does not fray during sampling and processing. The data in Table 4.2 show that RCF and Zorflex FM50K have higher resistivity and roughness than many of the new materials evaluated. This indicated some the new materials evaluated in FY 2016 are better suited for direct SIMS assay than the previous successful candidates. Ideally these materials would also be viable for bulk assay. Of the fabrics evaluated in FY 2016, several have promising properties and make them strong potential candidates. The fabrics that merit evaluation in the future are

- Carbon nanotube (CNT) fabric

- Carbon fiber twill weave (10.9 oz)

- Carbon fabric (3.5 oz)

- Graphite fabric (5.7 oz, and $10.9 \mathrm{oz})$,

- Metal-coated nylon (non-woven point bond, ripstop, and taffeta weaves).

Magnified images of the surface and weave of many of the preferred materials, and most shown in Table 4.2, are included in Appendix D. Although some of these materials are more expensive than traditional TexWipe TX304 sampling material, none of them are prohibitively expensive, given the labor and analytical cost associated with sample preparation. Further these are only promising preliminary material variants, not final suggested fabrics to be forwarded for full protocol evaluation. Variants of these materials will likely be the final candidate(s). Material types we have identified as effective and promising candidates for direct as SIMS assay (that should also be compatible with bulk ES methods) are discussed below.

\subsubsection{CNT Fabric and Graphene Coating Materials}

Carbon nanotubes (CNTs) fabric is an extremely durable, yet flexible material. It has the lowest electrical resistivity (highest conductivity of the carbon based materials). Its homogenous surface and minuscule fraying potential make it a great potential (must be tested) advancement to the current swipe sampling material. The metal content of CNT materials is typically low and the chemical inertness of the material allows residual metals/uranium to be cleaned with acid or based leaching solutions. Similar to CNTs in its physical structure (2D array of aromatic carbon molecules) and consequently has similar chemical robustness and good conductivity. Graphene coatings on a fabric (similar metals coatings on nylon and other fabrics) may have the ideal composite properties needed and deserve consideration in future efforts. 


\subsubsection{Carbon Fiber--Carbon Twill Weaves}

Carbon fiber fabric can be a highly conductive material with exceptionally low resistivity. Carbon fiber materials can be flat and have fairly low surface roughness. These materials have been found to have low backgrounds and can be cleaned with either acidic or basic methods (Table 3.6 and previous NA-22 work). These characteristics would make them potentially good candidates as conductive sampling materials. However, many of carbon fabrics evaluated had loose weaves and wide gaps between the threads, resulting in inconsistent pickup. Many of the carbon fiber fabrics would fray and shed material resulting in poor sampling integrity and complicating the analytical processing (especially for bulk processing of the samples). A carbon fiber fabric that provides a tighter weave, making it heavier and more durable, is essential.

Carbon fiber twill weave was found to be a very conductive material with good structural integrity, a fairly low surface roughness and notably low resistivity. The twill weave is a tightly woven diagonal pattern that provides stiffness and reduces fraying. The 19.8-oz twill weave material is stiff and there is not much “drape," which makes it ineffective as sampling material. The 10.9-oz carbon twill weave is preferred because of its flexibility and a lighter weave. This lighter-weight material should be explored because it would be a near match to the drape of physical characteristics of the approved cotton TexWipe 304 sampling material. The twill weave, on both the 10.9-oz and 19.8-oz carbon materials (and the cotton TexWipe 304) is extremely tight and provides extra reinforcement of fibers, which adds durability and reduces fraying. Carbon twill weave could be an excellent sampling material, however some treatment of the edges (stitching or epoxy seam) might be needed to prevent fraying if the material is to be used in bulk extraction as well as other flat, nonconductive nonfraying weaves that may become available.

\subsubsection{Graphite Fabric}

Graphite fiber is different from carbon fiber because it typically has been thermal treated and high temperatures and the carbon atom positioning is a hexagonal parallel lattice providing graphitic structure (and properties). The graphite fabric is a lightweight, yet highly durable material. It has a high tensile strength and low resistivity. It is a conductive material that can have good surface roughness values and a reasonably flat surface. However, for the graphite fabric to be a viable sampling material the correct weave material needs to be identified for improved structural integrity and flatness. To prevent fraying, some treatment of the edges (epoxy seam, stitching, etc.) likely would be needed.

\subsubsection{Metal-coated Fabric}

Metal-coated fabrics were a new class of material explored in FY 2016. These fabrics have found use in other safeguard applications and are commercially available in wide range of compositions. The metal coatings make the fabrics very conductive-almost as conductive as pure copper mesh (Table 4.2). These materials can also be very durable and resistant to fraying, depending upon the fabric used - an excellent example is metalized ripstop nylon. For consistency, in FY 2016 the research team evaluated metalized coatings all on nylon. The coated nylons can be very sturdy, flat materials with relatively low surface roughness — as can be seen from the metalized taffeta and ripstop values (Table 4.2). Unfortunately, and not entirely unexpectedly, high levels of uranium were observed in the metalized coating, making them unsuitable as sampling material. Large amounts of uranium were seen with bulk extraction (Table 3.6) from metalized cloth. High levels of ${ }^{235} \mathrm{U}$ and ${ }^{238} \mathrm{U}$ were directly observable on metalized nylon with 
SIMS. Despite good conductive, good flatness, and good mechanically properties (strong, limiting fraying, etc.) these metalized fabrics are presently unsuitable (as manufactured) as sampling materials due to the very high uranium backgrounds.

There are some methods where low uranium background metalized nylon (or other fabrics might be created. Metalized fabric with low uranium background could be made if ultra pure metals (e.g., the ultrapure copper PNNL produces for ultralow background nuclear detection) were used for the coating. However, this would not be economical or convenient. Purification could be achieved by chemically extracting the uranium from the commercially available materials. Acid leaching would remove metals indiscriminately, but a carbonate extraction (as discussed previously in Section 3) might be able to selectively clean the metal coating of uranium and reduce background to levels low enough for ultratrace assay. With the exception of a high-uranium backgrounds, selected metal-coated fabrics provide all the properties desired in a new swipe materials (for both bulk and SIMS assay): commercially available, strong, flat, durable, nonfraying and highly conductive. Carbonate extraction to reduce uranium levels in these almost ideal metal coated fabrics is a relatively simple experiment to conduct and merits consideration in future work. While not a commercial product metalized TexWipe 304 would be an interesting material to explore if research demonstrated that backgrounds could be reduced to an acceptable level.

\subsubsection{Summary}

In summary conductive fabric sampling materials were evaluated to enable direct SIMS assay with the intent to have them also remain compatible with bulk assay methods for ES samples. The desired properties of the materials are good conductivity (low resistivity), a high degree of flatness (low surface roughness), durability, and low uranium background. Previous efforts have shown some partially effective options; RCF (carbon tissue) and Zorflex FM50K (activated carbon cloth). FY 2016 work found promising materials offering potentially much better performance and meriting additional evaluation in the future. These promising polyfunctional (SIMS, bulk and maybe TIMS) sampling fabric candidates include:

- CNT fabric

- Carbon Fiber Fabrics (particular twill and other nonfraying flat weaves)

- Graphite fabric (with appropriate weaves)

- Treated (for background reduction) metalized fabrics

- Carbonate purified metalized nylon

- Graphene coated materials. 



\subsection{Task 3. Independent Technology Evaluation and Validation}

After the PNNL research team completed initial work on the rapid carbonate-peroxide uranium extraction chemistry they developed an initial procedure/protocol for rapid chemical extraction processing and delivered it, and selected sampling materials, to ORNL for independent evaluation selected against standard NWAL protocols for bulk ES sample assay. The ORNL analysis provides an independent validation of the strengths and limitations of the new materials and methods, as well as input for further optimization the new procedures. When the final analytical protocol is developed, ORNL will provide an independent gauge on the accuracy, precision, cost and timeliness of the new versus the standard analytical methods.

ORNL, work being led by Debra Bostick, will be providing a report independent of this PNNL report. The main results from ORNL are summarized as follows:

- The carbonate-peroxide uranium extraction chemistry can be effective and efficient for extraction of uranium from standard IAEA swipe materials (standards muslin/TexWipe TX304) and prototype materials (PTFE and carbon fiber) as well as providing a low background (in some cases lower than the standard TexWipe).

- PTFE swipes can provide very low uranium backgrounds and are particularly compatible with nondigestive extraction processes.

- The carbon fiber sampling materials provides low background with and without digestion processes. However, the structural integrity of carbon materials evaluated to date is not optimal; a denser carbon matrix with tighter weave might be very promising. Fabric produced from an activated carbon used in this study was too reactive with the current formulation of the leachate method. Other sources of activated carbon fabric may be more amenable to the PNNL extraction method.

- Uranium concentration using simple direct "wet” sample introduction into a single sector ICP-MS appears to be similar between the NWAL and new protocols (as measured by ICP-MS) for determination of uranium concentration. However, the high salt content of the sample leachate plugs MC-ICP-MS systems equipped with and without desolvator introduction systems. Until the dissolved solids content of the leachate is reduced to less than 0.005 percent or $5 \mathrm{E}-04 \mathrm{M}\left(\mathrm{NH}_{4}\right)_{2} \mathrm{CO}_{3}$, repeatable accurate isotopic analysis is not possible using direct sample addition into a MC-ICP-MS.

- Using a modified procedure rapid sensitive uranium analysis is still possible using the carbonate extraction process. The carbonate leachate is simply evaporated and the carbonate residue ignited and reduced in a furnace. Similar to portions of the existing NWAL method, the residue is then acidified, cleaned up with microcolumn, and run on ICP-MS with good results. The significant difference is the 15 days required to digest and chemically process a swipe sample for uranium by the standard ORNL NWAL procedure is reduced to three days using the new procedure. 



\subsection{Sample Preparation}

Standards are essential for any accurate analytical process and are particularly important for measurements having nuclear security and international treaty implications. Generation of standards for uranium enrichment measurement validation is problematic because of the tendency of $\mathrm{UF}_{6}$ to sublime and react with moisture and substrates. Standards generation has an additional challenge of making and handling quantitative mixed uranium isotope standards; the ratio of ${ }^{235} \mathrm{U}$ to ${ }^{238} \mathrm{U}$, for instance, is a key metric for monitoring uranium enrichment activities. Present IAEA analytical protocols avoid the use of unstable and unavailable $\mathrm{UF}_{6}$ standards (Vogt et al. 2001). However, the ability to generate uranium standards, particularly at low mass levels and with controllable isotopic ratios, is essential for analytical systems calibration and validation, analytical protocol development and evaluation.

PNNL recently reported a convenient method for the generation of volatile uranium hexafluoride $\left(\mathrm{UF}_{6}\right)$ from solid uranium oxides and other uranium compounds, followed by uniform deposition of low levels of $\mathrm{UF}_{6}$ onto sampling coupons (McNamara et. al. 2016). Under laminar flow conditions, $\mathrm{UF}_{6}$ is shown to interact with surfaces within a fixed reactor geometry to a highly predictable degree. McNamara et al. demonstrated the preparation of uranium deposits that range between approximately 0.01 and $500 \mathrm{ng} \mathrm{cm}^{2}$. These data suggest the method can be extended to creating depositions at the subpicogram $\mathrm{cm}^{-2}$ level. The process is shown graphically in Figure 6.1. The isotopic composition of the deposits can be customized by selection of the uranium source materials and the research team demonstrated a layering technique whereby two uranium solids, each with a different isotopic composition, could be employed sequentially to form mixed composite samples.

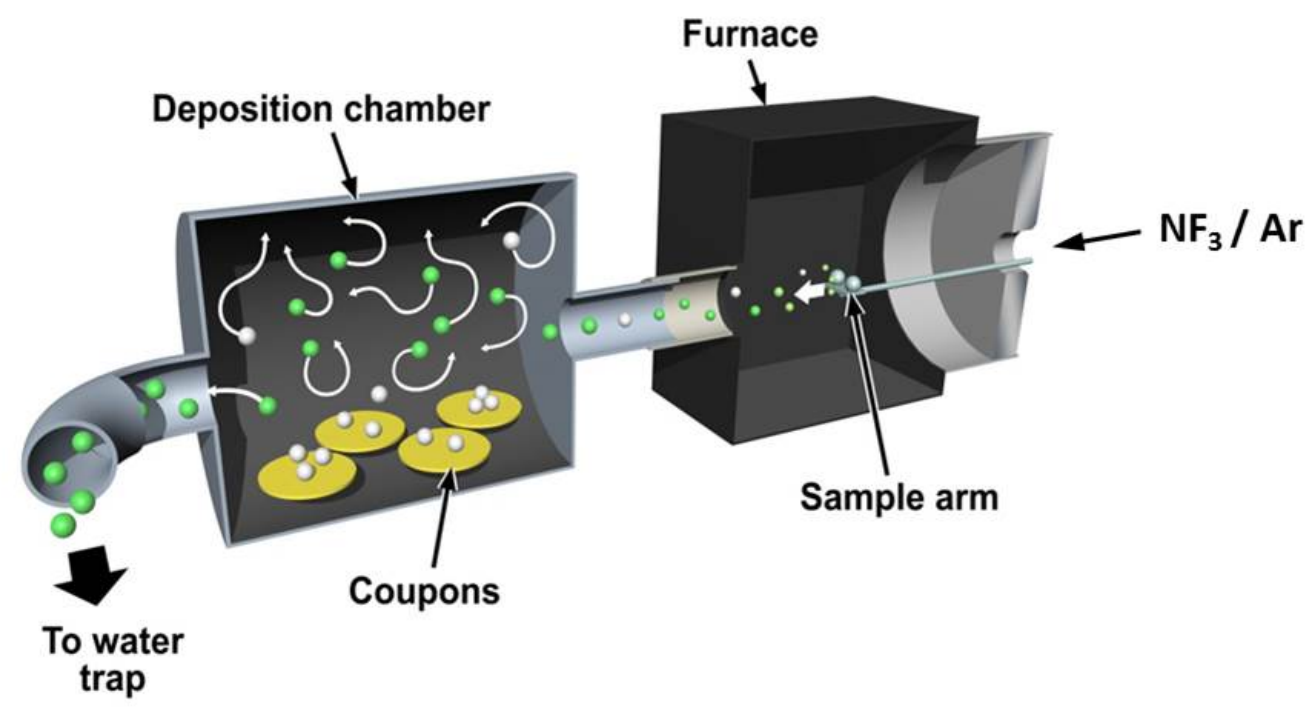

Figure 6.1. Experimental Apparatus for Deposition of $\mathrm{UF}_{6}$ onto Coupons. The uranium source material is added to a pan at the end of the sample arm. An influent mixture of $\mathrm{NF}_{3}$ and Ar converts $\mathrm{UO}_{2}$ to $\mathrm{UF}_{6}$ (white molecules). The $\mathrm{UF}_{6}$ is transported through a conduit by the Ar carrier gas flow (green atoms). Next, the $\mathrm{UF}_{6} / \mathrm{NF}_{3} / \mathrm{Ar}$ stream enters the deposition chamber. $\mathrm{UF}_{6}$ is deposited on the surfaces of the deposition chamber. This includes deposition onto the sample coupons placed on a sample tray (tray not shown). Residual $\mathrm{UF}_{6}$ flows from the deposition chamber and is captured by a water trap. 
The method described above deposits $\mathrm{UF}_{6}$ in the form of small particles-the morphology and size of which are dependent upon conditions_-across the surface of a sample and the materials is rapidly chemically converted to uranium oxide fluorides species rapidly. The $\mathrm{UF}_{6}$ deposits have been demonstrated to be stable toward changes in atmospheric and vacuum conditions. The $\mathrm{UF}_{6}$ deposition can be directly upon the sample (or sampling material as shown in Fig. 6.2) or upon material to be sampled (i.e., mineral dust as shown in Figure 6.3). The methods have the ability to form samples that are chemically, physically and isotopically matched to specific desired enrichment scenarios. This technology was used to prepare a range of samples for this program and the technology has direct application to the development of unique analytical standards for nuclear safeguards and forensics.

Using the described method the research team prepared a range of realistic enrichment samples with accurate isotopic ratios, particle sizes, and activity levels for both SIMS and bulk sample assay, and found the following:

- SIMS and ICP-MS data showed isotopic ratios very similar to the starting material used in the method to make the sample materials.

- Extremely low-level samples (10-200 cpm) could be made that provide realistic ultra-trace level samples and enabled handling and shipping without significant radiological concerns.

- Samples could be made with a wide range of isotopic ratios, ranging from natural to HEU.

- Samples could be made with multiple types of particles (i.e., natural, LEU, 20 percent and HEU).
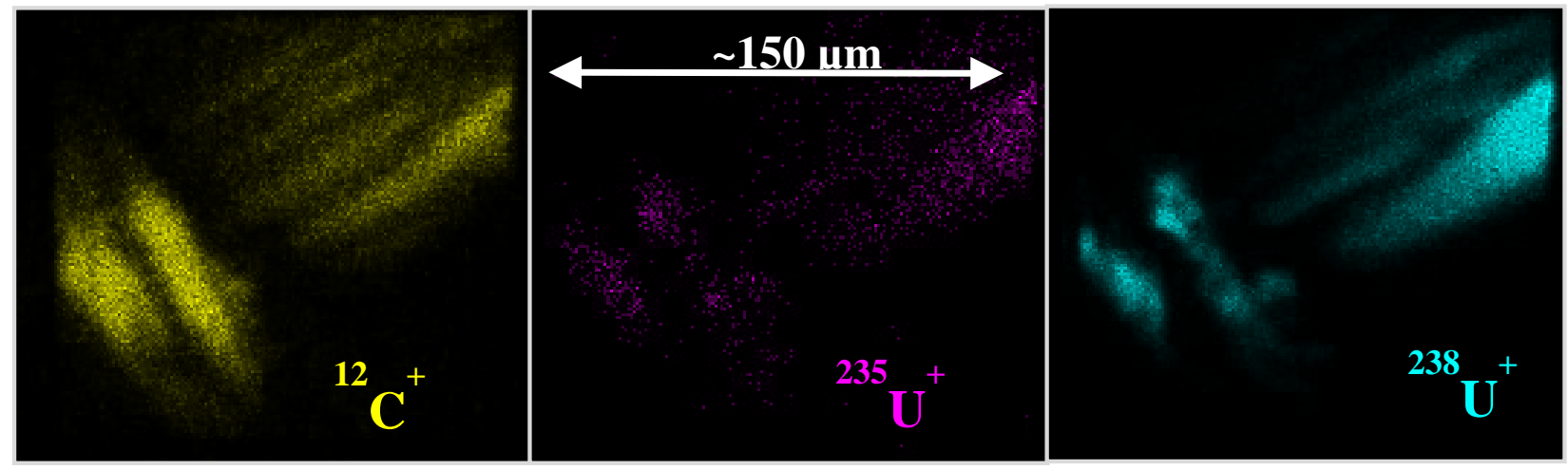

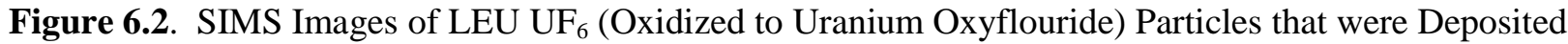
Directly upon Carbon Fiber Sampling Cloth. The carbon ion (yellow, left) image clearly shows fibers of the sampling cloth. The uranium ion images show the material is uniformly distributed in very small particles across the surface. 


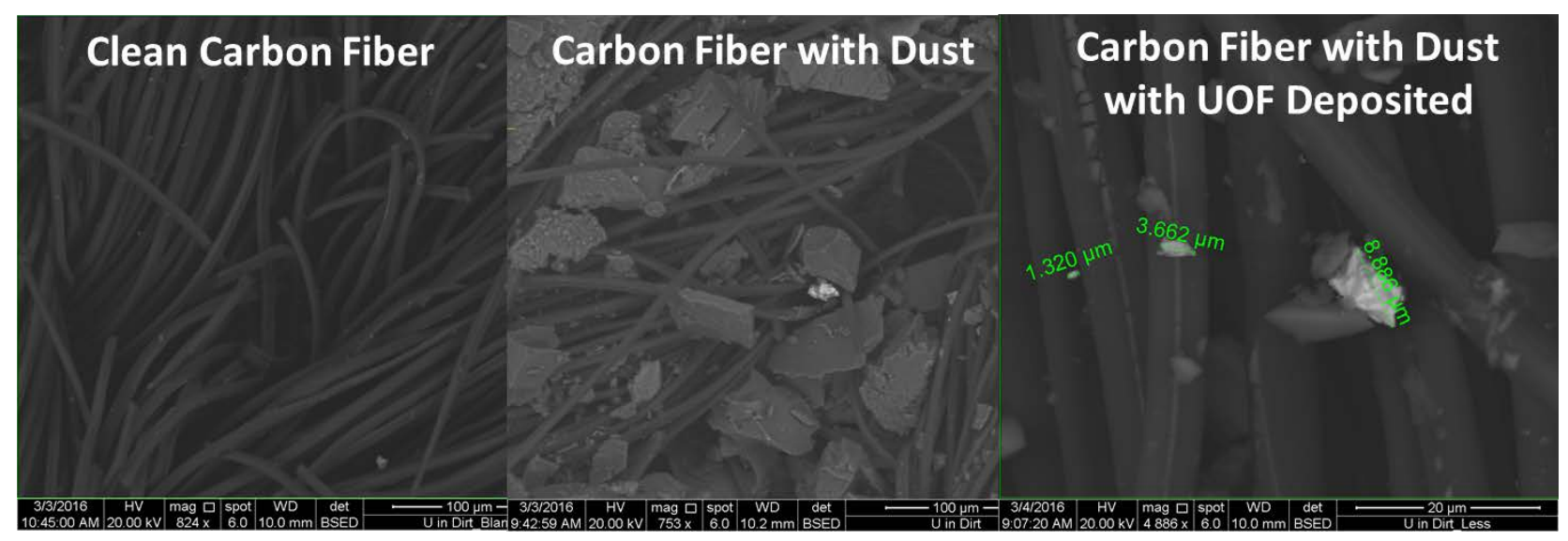

Figure 6.3. An Example of Dust Samples on Carbon Fiber Sampling Cloth. The UOF particles (brighter particles) on the right were generated with the PNNL $\mathrm{UF}_{6}$ vapor generation system which allows controlled deposition of ultra low levels of UOF particles with specific isotope ratios. 



\subsection{Future Work}

The International Atomic Energy Agency (IAEA) relies upon laboratory analysis of environmental samples (typically referred to as "swipes") collected during on-site inspections of safeguarded facilities to support the detection and deterrence of undeclared activities. Unfortunately, analytical processing and assay of the samples is slow and expensive. Rapid, effective, and simplified analytical protocols that provide certified results would improve data timeliness at lower costs (principally in the form of reduced labor), while maintaining or improving sensitivity and efficacy.

To and address these safeguard needs in FY 2016 PNNL matured and demonstrating improved analytical methods for streamlined analysis of environmental samples. Improvements for both bulk and particle analysis have been explored. To facilitate continuity and adoption, the new sampling materials and processing methods are designed to be compatible with existing IAEA protocols for ES analysis. PNNL collaborated with ORNL, which performed independent evaluation and validation of the new bulk analysis methods and compared performance to traditional NWAL protocol. As detailed in this report significant advances were made by PNNL and ORNL in FY 2016. Additional focused efforts are needed to finalize the materials and methods that would provide finished analytical methods for evaluation to the larger safeguards community.

Future effort to complete the technology development and provide finalized protocols that will provide faster and more cost effective laboratory safeguard sample analysis include:

- Optimize and finalize materials and methods for (bulk) ICP-MS ES analysis.

- Extraction Chemistry. The carbonate-peroxide chemistry has been demonstrated to be effective and faster than existing methods. Final efforts are needed to optimize and define best extraction conditions for a robust and simple process. The method needs to be evaluated for a range of uranium sources and for challenging “dirty” samples. The finalized method will need to be formalized into a specific analytical protocol.

- Sampling Materials. Effective alternative with low backgrounds have been demonstrated with Teflon (PTFE) and carbon fiber-based swipes. Final material composition and commercial sources must be identified and performance demonstrated. Ideally the new material would support improved SIMS as well as TIMS.

- Evaluation. New chemical methods and sample materials will need to be evaluated by an independent NWAL facility such as ORNL, and the finalized protocol(s) need to be tested on realistic samples.

- Optimize and finalize materials and methods for direct SIMS ES analysis of particles.

- Sampling Materials. Effective conductive materials that enable direct SIMS assay of ES swipes have been demonstrated (metalized fabric carbon fiber materials). Final material composition and commercial sources must be identified and their performance must be demonstrated. Ideally the new material would support accelerated bulk ES methods as well as TIMS.

- Sample Screening. Explore and validate sample screening methods to rapidly identify locations on the swipe sample that merit direct SIMS assay (eliminating lengthy searching for particles of interest). This will include integration of improved SIMS searching methods to quickly identify regions of interest for detail analysis. 
- Sample Mounting. Finalize design and demonstrate sample holder to enable simple and rapid mounting of swipe samples for direct SIMS assay. The sample mount will support automation, integration, and cross correlation with other screening methods, and improve imaging performance. Methods to make the sample mounts cheap and disposable (such as 3D-printed conductive plastics) will support higher sample throughput and cost.

- Thermal Ionization Mass Spectrometer (TIMS)

- To date streamlining and accelerating TIMS ES analysis has not been explored as part of the streamlining effort. Leveraging previous NNSA investments on developments and improvements demonstrated for bulk and SIMS ES analysis would be advantageous-particularly in the light of the extensive sample preparation efforts required for TIMS analysis. Discussions with NWAL TIMS staff at PNNL indicate a potential for streamlining/improving the TIMS process with the developed methods.

\section{Improved Field Screening Assay.}

Efforts to date have been focused specifically upon improving and accelerating laboratory NWAL methods. The Additional Protocols support adaptive sampling and in-field assay and the IAEA has expressed an interest in improving on-site analysis capabilities with a particular focus on tools that could affect the course of their inspections at nuclear facilities. Further, assay in the field avoids the increasingly complex and problematic issues involved in shipping radioactive samples.

Analytical improvements in field assay are directly aligned with the IAEA Long Term R\&D plan; specifically Sections 2.2 and 2.6. The effort provides solutions to a number of the major challenges identified by the Global Safeguards (GS) program including enabling a “Connected Inspector” (by providing inspectors with new methods and materials to better enable on-site inspection capabilities). Significant work has gone into development of field assay instruments (as noted in sections 7 and 8 of IAEA Safeguard Techniques and Equipment; 2011; No. 1, Rev 2). Some "fieldable" radiochemical methods such as those used by the COMPUCEA (Erdmann 2009, Erdmann 2009, Erdmann 2008, IAEA 2010 CN-184/316) have garnered recent use and notable mention. Leveraging the previous NNSA investments and capabilities developed for streamlined and accelerated laboratory analysis would provide significant improvements (speed, simplicity, sensitivity) the IAEA’s field screening capabilities. 


\subsection{References}

Addleman RS, JC Anheier, CA Barrett, JC Carter, AM Casella, W Chouyyok W, BK McNamara, BE Naes, MJ O'Hara, KB Olsen, RJ Speakman, and DC Willingham. 2016. Improved Collection and Measurement of Safeguards Signatures Using Engineered Materials and Processes. PNNL-25403, Pacific Northwest National Laboratory, Richland, Washington. (Limited Distribution)

Erdmann N, P Amador, P Arboré, H Eberle, K Lützenkirchen, H Ottmar, H Schorlé, P van Belle, F Lipcsei, P Schwalbach, and R Gunnink. 2009. "COMPUCEA: A high-performance analysis procedure for timely on-site uranium accountancy verification in LEU fuel fabrication plants.” ESARDA Bulletin, 43:30-39.

Erdmann N, H Ottmar, P Amador, H Eberle, H Schorlé, and P van Belle. 2009. Validation of COMPUCEA 2nd Generation, JRC-ITU-TN-2008/37, European Commission, Joint Research Centre, Institute for Transuranium Elements, Karlsruhe, Germany.

Erdmann N, N Albert, P Amador, P Arboré, H Eberle, K Lützenkirchen, H Ottmar, H Schorlé, P van Belle, F Lipcsei, P Schwalbach, S Jung, and R Lafolie. 2010. COMPUCEA 2nd generation performance evaluation. IAEA-CN-184/316. International Atomic Energy Agency, Vienna, Austria.

IAEA - International Atomic Energy Agency. 2011. IAEA Safeguard Techniques and Equipment, 2011 Edition. International Verification Series No. 1, Rev 2. International Atomic Energy Agency, Vienna, Austria.

IAEA - International Atomic Energy Agency. 2013. IAEA Department of Safeguards Long-Term R\&D Plan, 2012-2023. STR-375, International Atomic Energy Agency, Vienna, Austria.

Matthias Kohler , Gary P. Curtis ,David E. Meece , and James A. Davis “Methods for Estimating Adsorbed Uranium(VI) and Distribution Coefficients of Contaminated Sediments” Environ. Sci. Technol., 2004, 38 (1), pp 240-247. DOI: 10.1021/es0341236

McNamara BK, MJ O’Hara, AM Casella, JC Carter, RS Addleman, and PJ MacFarlan. 2016. “Uniform deposition of uranium hexafluoride $\left(\mathrm{UF}_{6}\right)$ : Standardized mass deposits and controlled isotopic ratios using a thermal fluorination method.” Talanta, 154:219-227. http://dx.doi.org/10.1016/j.talanta.2016.03.054

Smith, S.C., Peper, S.M., Douglas, M. et al. J Radioanal Nucl Chem (2009) 282: 617. doi:10.1007/s10967-009-0182-8

Vogt S, P Zahradnik, D Klose, and H Swietly. 2001. Bulk Analysis of Environmental Swipe Samples. IAEA-SM-367/10/06, International Atomic Energy Agency, Seibersdorf, Austria. http://wwwpub.iaea.org/MTCD/publications/PDF/ss-2001/PDF\%20files/Session\%2010/Paper\%2010-06.pdf

Willingham D, BE Naes, PG Heasler, MM Zimmer, CA Barrett, RS Addleman. 2016. “Image segmentation for uranium isotopic analysis by SIMS: Combined adaptive thresholding and marker controlled watershed approach.” Journal of Vacuum Science \& Technology B 34(3), 03 H106. 

Appendix A

Safeguards Technology Fact Sheet, Streamlined Analysis of Environmental Samples 



\section{Safeguards Technology Fact Sheet ONvs:}

\section{Streamlined Analysis of Environmental Samples (ES)}

\section{Summary}

PNNL explored developments of two streamlined methods to prepare environmental samples for bulk and particle analysis by the primary analytical methods used respectively by the International Atomic Energy Agency (IAEA): inductively coupled plasma mass spectrometry (ICP-MS) and secondary ion mass spectrometry (SIMS). To facilitate continuity and adoption, the new sampling materials and processing methods will be compatible with existing IAEA protocols for environmental sample (ES) analysis. PNNL collaborated with Oak Ridge National Laboratory (ORNL), which performed independent bulk analysis of samples prepared by the streamlined process and the traditional IAEA's Network of Analytical Laboratories (NWAL) protocol to validate the methods.

\section{Background}

The IAEA relies upon laboratory analysis of environmental samples (typically surface "swipes") during on-site inspections of safeguarded facilities to support the detection and deterrence of undeclared activities. Unfortunately, chemical processing and assay of the samples is slow using today's methods, which can significantly delay reporting results and lead to sample backlogs. Faster, simpler sample processing and analysis methods are needed to provide certified results with improved timeliness and at reduced costs, while maintaining or improving sensitivity and efficacy. This task addresses that recognized need.

\section{Func tional Elements}

- $\quad$ Faster and more efficient sample processing with regards to handling and labor

- Maintained or improved sensitivity and efficacy to quantifying key analytical markers

- Complete compatibility with existing IAEA protocols for ES analysis

- Methods validated independently (i.e., by a member of NWAL at another site)

\section{Operational Description}

PNNL evaluated and demonstrated advanced sampling materials and processing methods to improve and accelerate laboratory analysis of environmental samples in two areas:

1. Optimize materials and methods for ICP-MS ES analysis (see Figure 1, left side) and demonstrate previously developed sample preparation protocols that accelerate and streamline the bulk (as opposed to particle-specific) assay of surface/swipe samples using the IAEA's existing ICP-MS instrumentation. As graphically shown on the left side of Figure 1, this effort will streamline the ICP-MS sample preparation. FY 2016 efforts focus on ES collected for UF 6 process monitoring but the methods were also successfully applied to uranium oxides, nitrates, and natural minerals. PNNL collaborated with ORNL staff to take the initial materials and procedures generated at PNNL and independently evaluate them against standard NWAL protocols. ORNL analysis validated the strengths and identified some problems remaining to be resolved with the new materials and procedures. Subsequent work will enable development of finalized protocols and subsequent competitive evaluation to gauge the advantages of the new versus the current analytical methods relative to accuracy, precision, speed and cost.

2. Optimize materials and methods for direct SIMS ES particle analysis. (See Figure 1, right side.) The effort explored development of analysis protocols and commercial sources of sampling materials that enable direct SIMS assay of surface/swipe samples with minimal sample preparation. Improved samples mounts, enhanced automated searching for enriched particles, novel conductive sample materials with low backgrounds, and direct accurate isotopic assay for enriched uranium particles on ES swipes samples have 
been demonstrated. Further optimization and integration of individual component improvements are still needed to provide a new cohesive protocol for faster and simpler SIMS of ES samples.



Figure 1. A graphical comparison between the existing and proposed methods for preparation and analysis of ES. The ICP-MS provides bulk sample measurement while the SIMS provides the capability to assay specific particles on a sample. The methods and materials under development provide simpler, faster, and more costeffective methods for measurement of safeguard environmental samples.

\section{FY 2016 Status}

FY 2016 focused on optimizing and validating previously developed techniques for improvement of both bulk and particle uranium ES measurements. PNNL completed initial laboratory evaluations and preliminary procedures for improved ICP-MS and SIMS analysis. PNNL initiated technology commercialization efforts. ORNL completed an independent peer review of initial proposed bulk analysis methods and proposed improvements. ORNL found the extraction process was effective but needed to be optimized for NWAL systems

\section{References}

1. R. S. Addleman, et al., "Improved Collection and Measurement of Safeguards Signatures Using Engineered Materials and Processes," 2014 Annual report for NA-22, WMS Project PL13-GS, Signatures-PD2La.

2. McNamara, B. K.; O’Hara, M. J.; Casella, A. M.; Carter, J. C.; Addleman, R. S.; MacFarlan, P. J., Uniform deposition of uranium hexafluoride (UF6): Standardized mass deposits and controlled isotopic ratios using a thermal fluorination method. Talanta 2016, 154, 219-227.

3. Willingham, D.; Naes, B. E.; Heasler, P. G.; Zimmer, M. M.; Barrett, C. A.; Shane Addleman, R., Image segmentation for uranium isotopic analysis by SIMS: Combined adaptive thresholding and marker controlled watershed approach. Journal of Vacuum Science \& Technology B 2016, 34 (3), $03 \mathrm{H} 106$.

Additional patents and publications pending

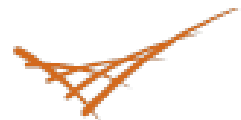




\section{Appendix B}

\section{Extraction Method Details for Bulk Environmental Sample}

Swipes 



\section{Appendix B}

\section{Extraction Method Details for Bulk Environmental Sample Swipes}

A select amount of the uranium particles $\mathrm{U}_{3} \mathrm{O}_{8}$ and $\mathrm{UO}_{2} \mathrm{~F}_{2}$ were suspended in a liquid using mild sonication. Concentration of each colloidal suspension was maintained and validated at $0.1( \pm 0.05) \mathrm{mg} / \mathrm{mL}$. Approximately $1.5 \mu \mathrm{g}$ of uranium particulates was deposited to small swatches ( $2.25 \mathrm{~cm}^{2}$ in size) of each swipe material. The deposited swipe material was allowed to air dry completely overnight before extraction. Each swatch of deposited material was then placed in plastic vials with $5 \mathrm{~mL}$ of extracting solution. The vials were capped and shaken at $100 \mathrm{rpm}$ for 18 hours. The swipe material was then removed and the solutions were diluted with $\mathrm{HNO}_{3}$ before uranium analysis with multi-collector inductively coupled plasma mass spectrometry (MC-ICP-MS).

Note, in some spots extraction values given are over 100 percent (with standard deviation) because the suspended uranium particles were deposited on the swipe manually. In some cases slightly larger particles (by particle size and number) may have been deposited on the swipe than those in the control bottles, which resulted in slightly different concentrations of uranium after the particle was dissolved in the extractant fluid. Therefore, final extraction in the process of some spots had slightly higher than 100 percent extraction. 



\section{Appendix C}

Measurement Methods for Potential SIMS Sampling Materials 



\section{Appendix C}

\section{Measurement Methods for Potential SIMS Sampling Materials}

\section{C.1 Resistivity Measurement}

The resistivity of the conductive sample materials was measured by using a four-point probe and electrometer. Each fabric was placed underneath the four-point probe apparatus with a nonconductive material underneath to prevent inclusion of the base of the apparatus into the measurement reading. A voltage was induced within the two inner probes when the current was applied through the two outer probes. The LabTracer 2.9 software application was used to adjust the current and various currents were applied to each material until the graph within the software application displayed a straight line. The straight line signaled that a valid resistivity could be calculated for the material. Four sets of voltage and current measurements were made for each current through LabTracer 2.9. The average voltage and current from the measurements were found to calculate the sheet resistivity, $R_{S}=4.54 \frac{\mathrm{V}}{\mathrm{I}}$. Then, sheet resistivity was multiplied by the thickness of the material to get the resistivity of the material, $\rho=R_{S} T$ with units $(\Omega \cdot \mathrm{cm})$.

\section{C.2 Roughness Measurement}

The surface profiles of the fabric materials were measured by a Bruker Counter GT-K1 white light inferometer. Green light was used due to its increased sensitivity over white light. The noise threshold was set to the minimum value, 0.001 , to ensure that all fringes from the material were collected.

All profile images were extracted from the Bruker Vision64 software as an ASC file and converted using a MATLAB script for processing. Data processing occurred to remove any missing points prior to extracting the average surface roughness (Rms, $\mu \mathrm{m}$ ) from the statistical quantities measurement tool. When multiple inferometery profiles were collected of the same spot, the profile with the least amount of missing data was selected for further analysis. 



\section{Appendix D}

High Magnification Images of Potential Sampling Fabrics that May Enable Direct SIMS 



\section{Appendix D}

\section{High Magnification Images of Potential Sampling Fabrics that May Enable Direct SIMS}

In FY 2016 the research team explored conductive swipe materials to determine materials that could enable direct SIMS measurement without the labor-intensive sample preparation steps. Magnified images of many of the preferred materials found in this effort are included in this Appendix. 



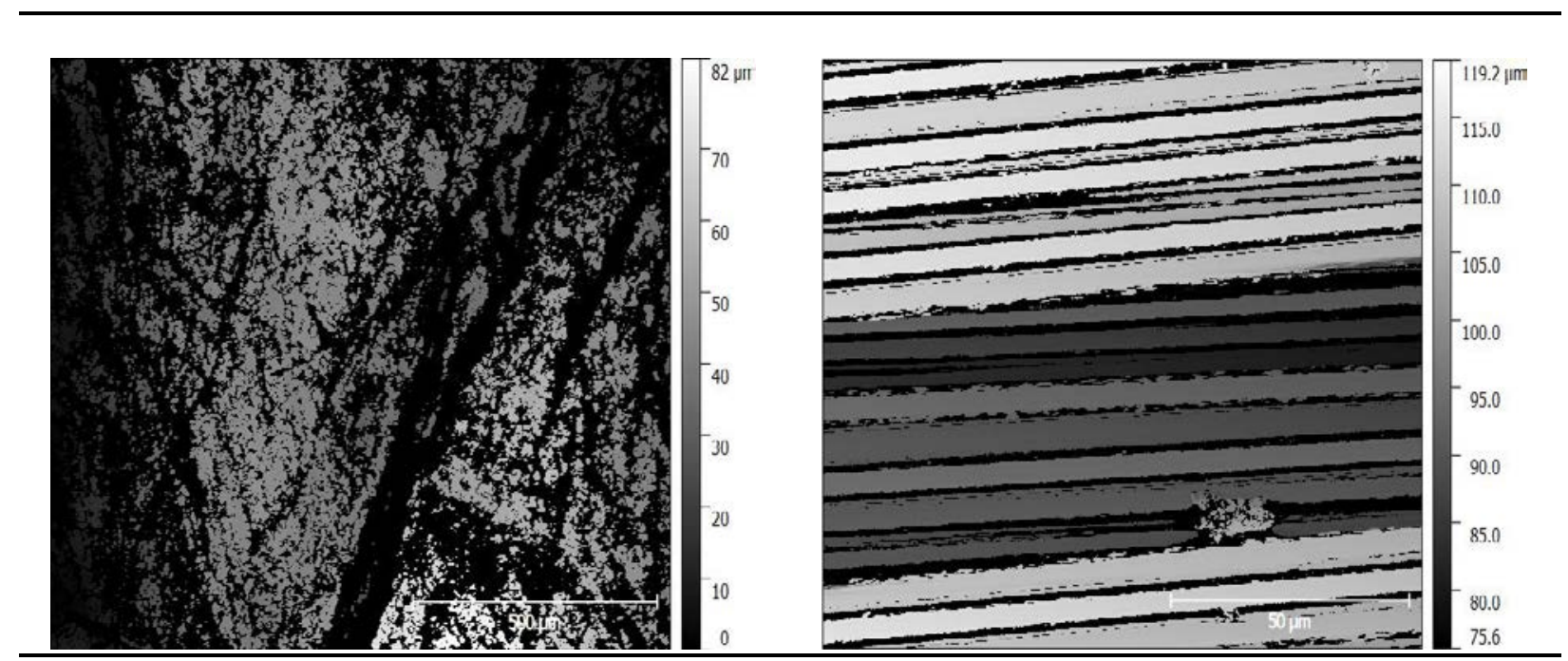

CNT Coated Fabric (5x)

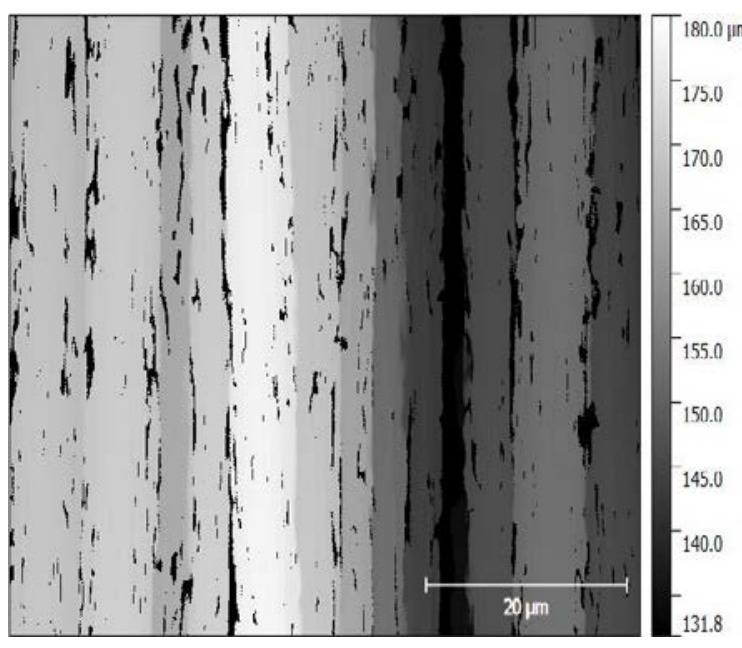

Carbon Fabric 3.5 oz (50x)

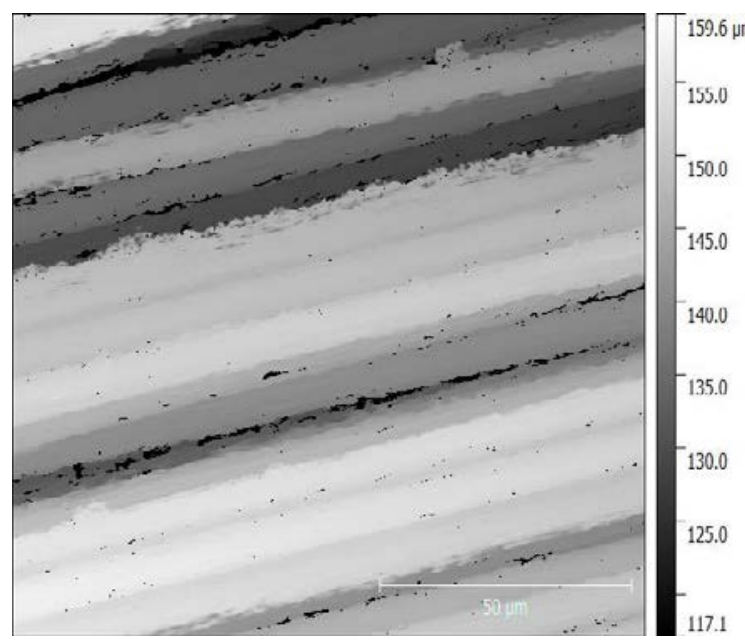

Graphite Fabric 5.7 oz (50x)

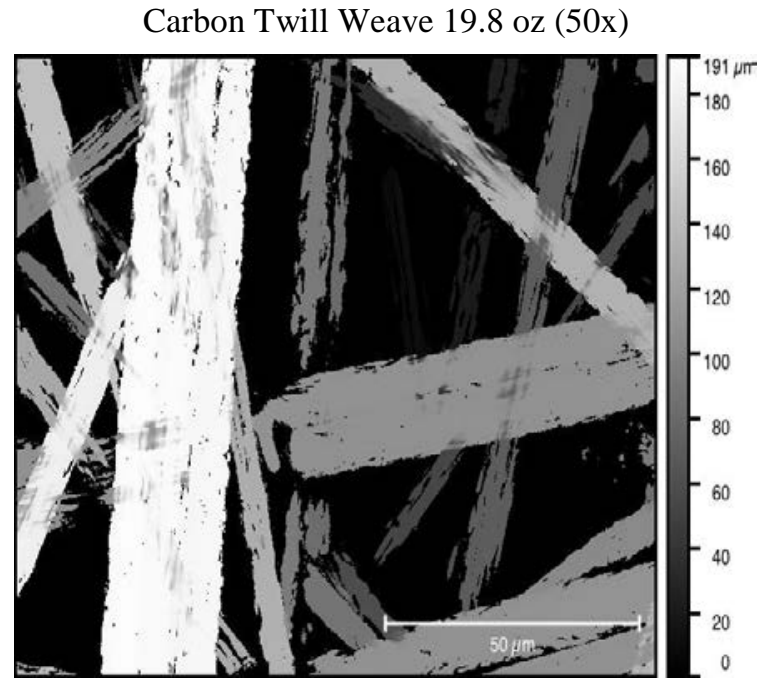

Carbon Tissue (RCF) (50x)

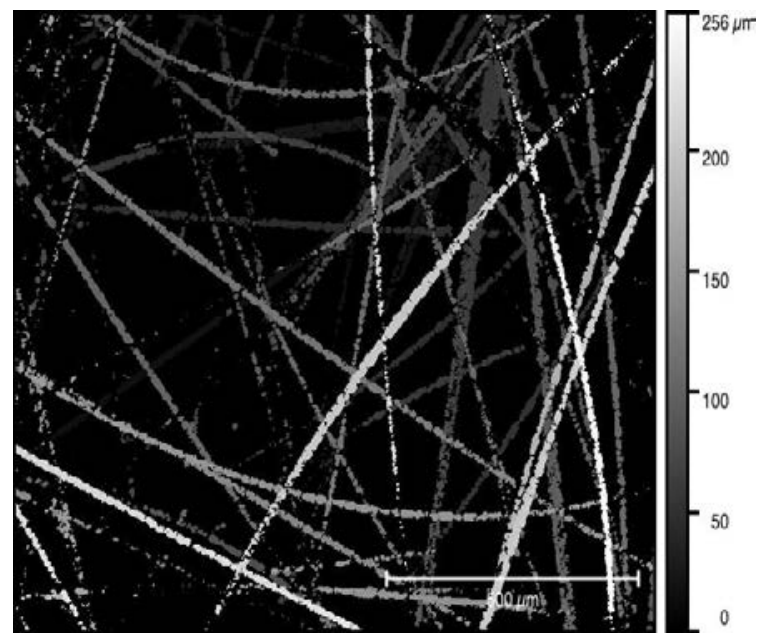

Graphite Veil .2 oz (5x) 


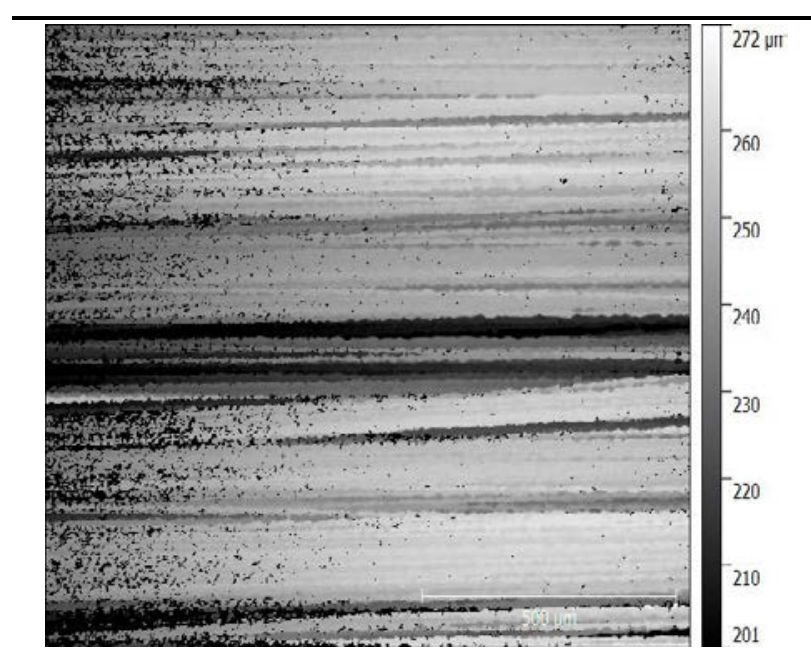

Graphite Fabric 10.9 oz (5x)

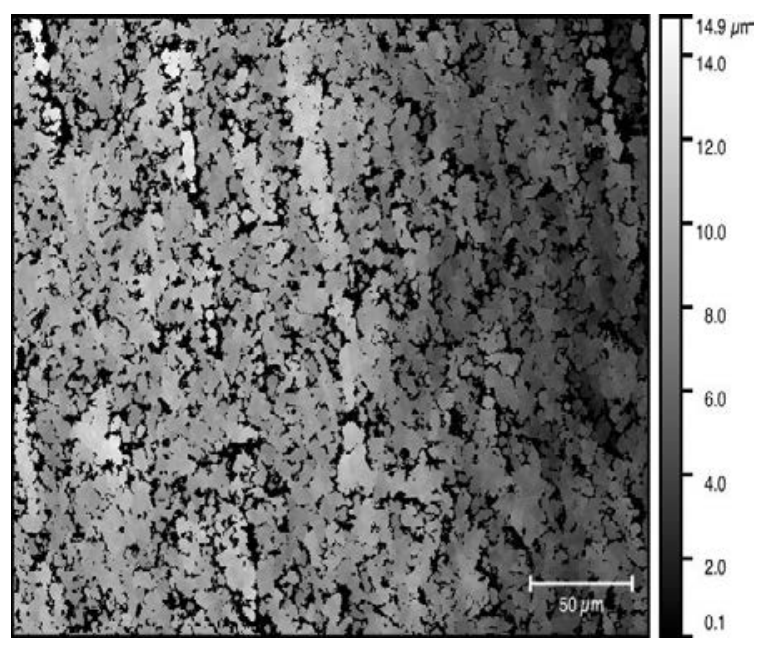

Activated Carbon Fiber Cloth

(Zorflex double weave) (50x)

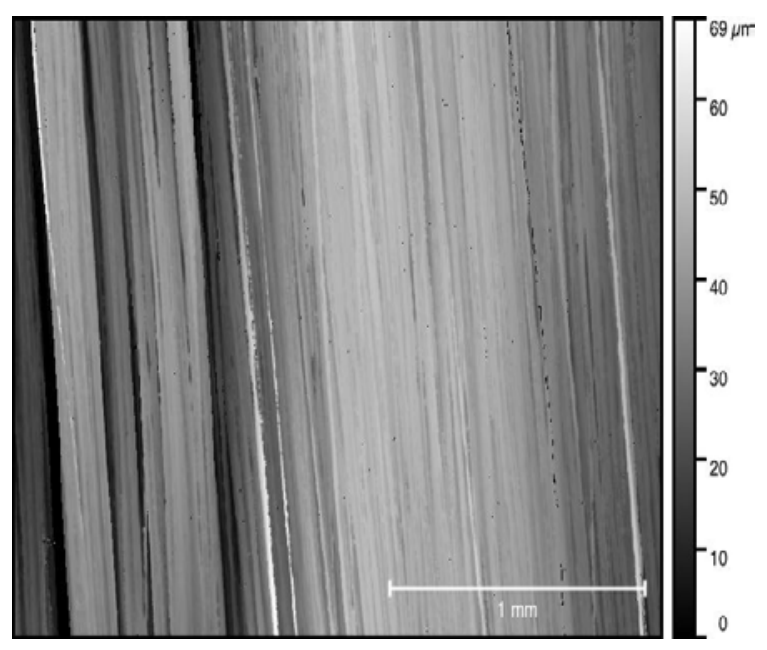

Unidirectional Carbon 9 oz (5x)



Carbon Twill Weave 10.9 oz (50x)

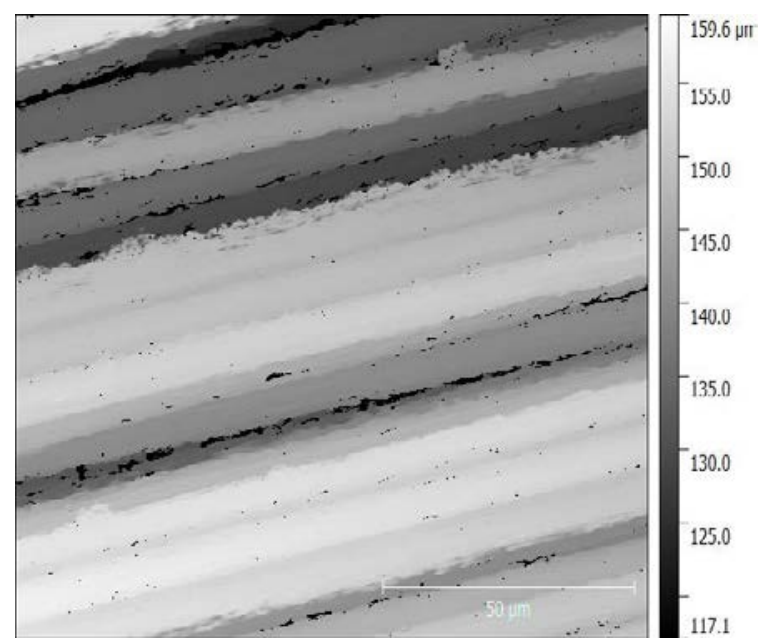

Graphite Twill Weave 5.7 oz (50x)

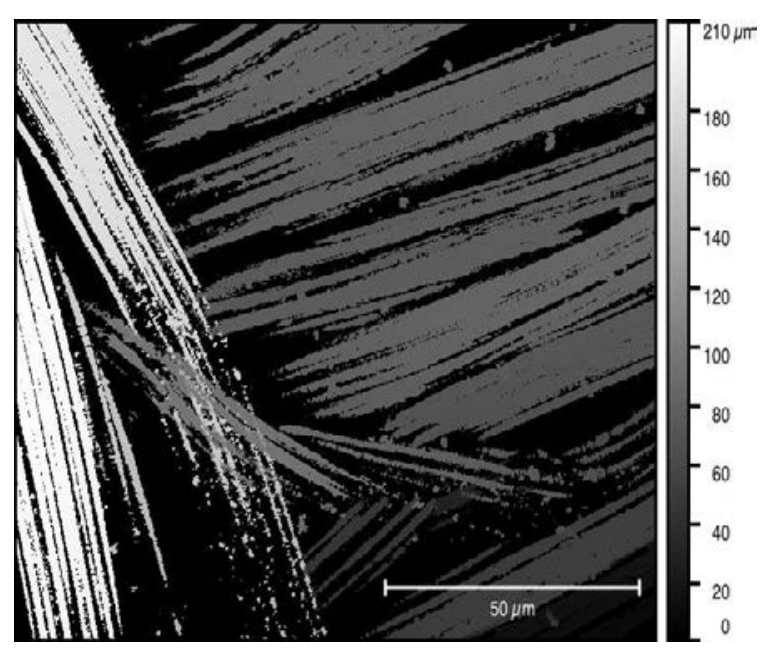

Activated Carbon Fiber Cloth

(Zorflex single weave) (50x) 


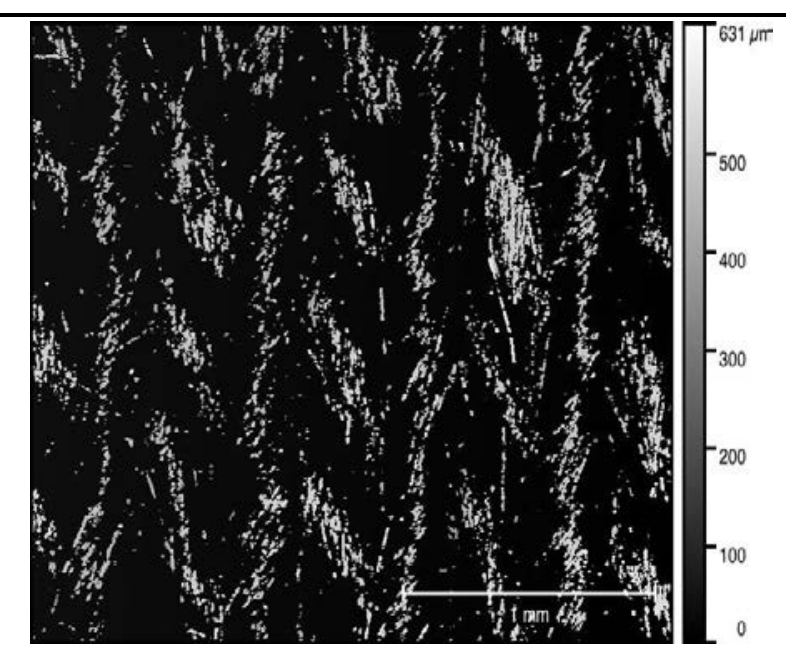

Activated Carbon Cloth (Zorflex FM50K) (5x)

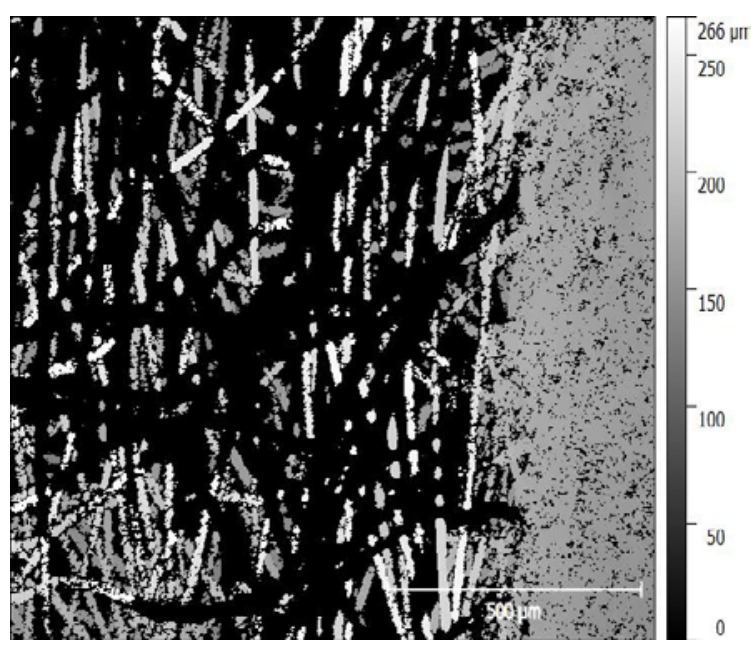

Ni Ag Coated Non-Woven Point Bond (5x)

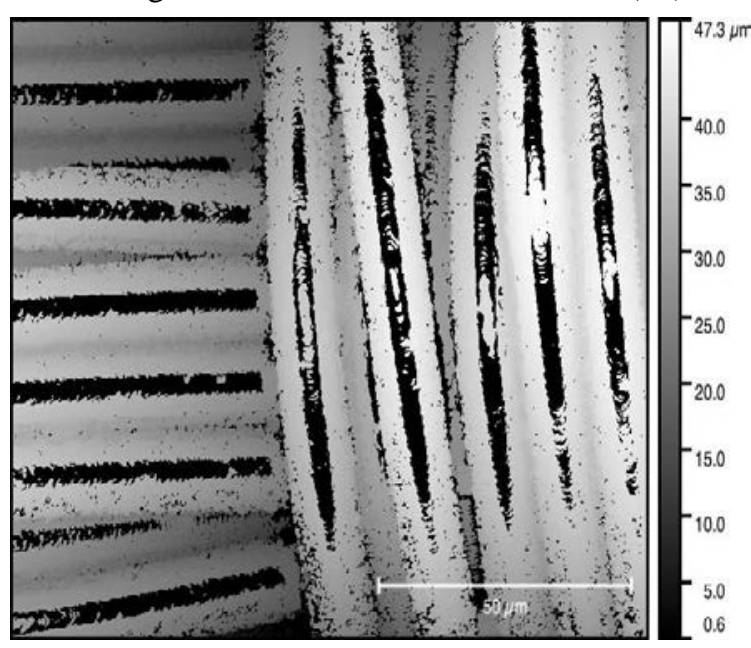

Ni Ag Coated Nylon Taffeta (50x)

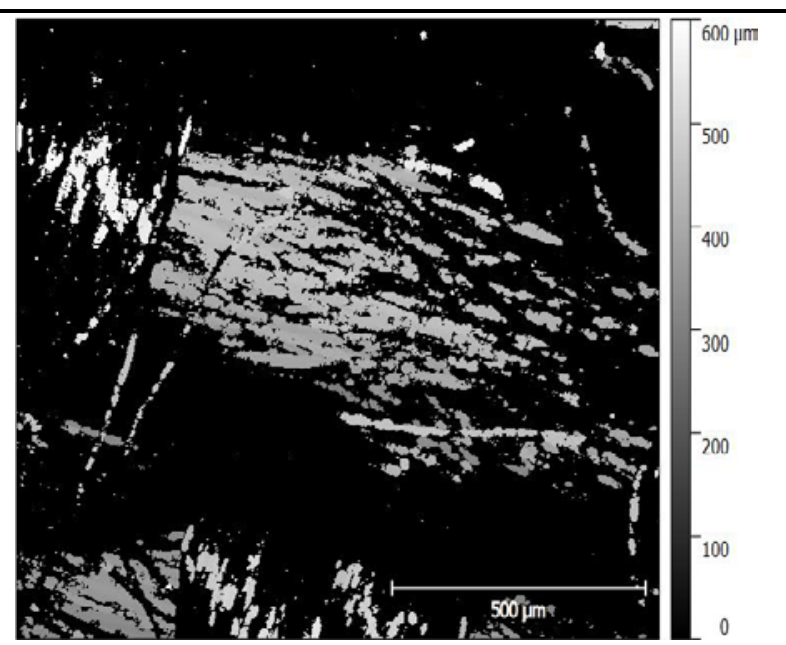

Activated Carbon Cloth (Zorflex VB+) (5x)

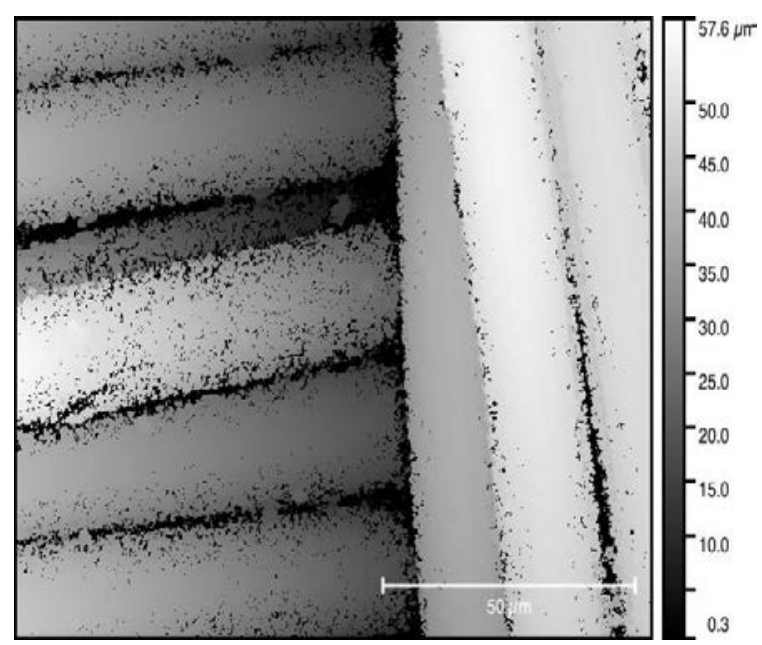

Ni Ag Coated Nylon Ripstop (50x)

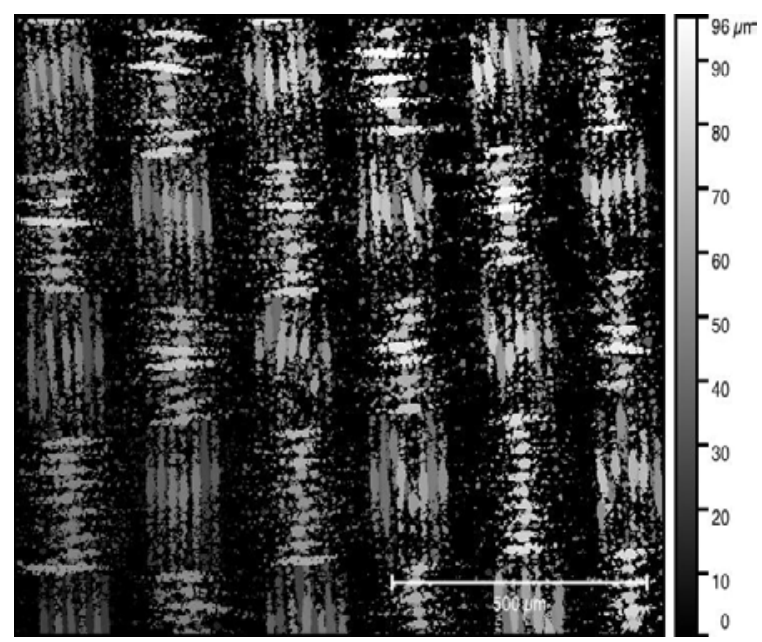

Ag Coated Nylon Ripstop (5x) 




Ni Ag Coated Non-Woven (5x)



Ag Coated Tricot Knit (5x)

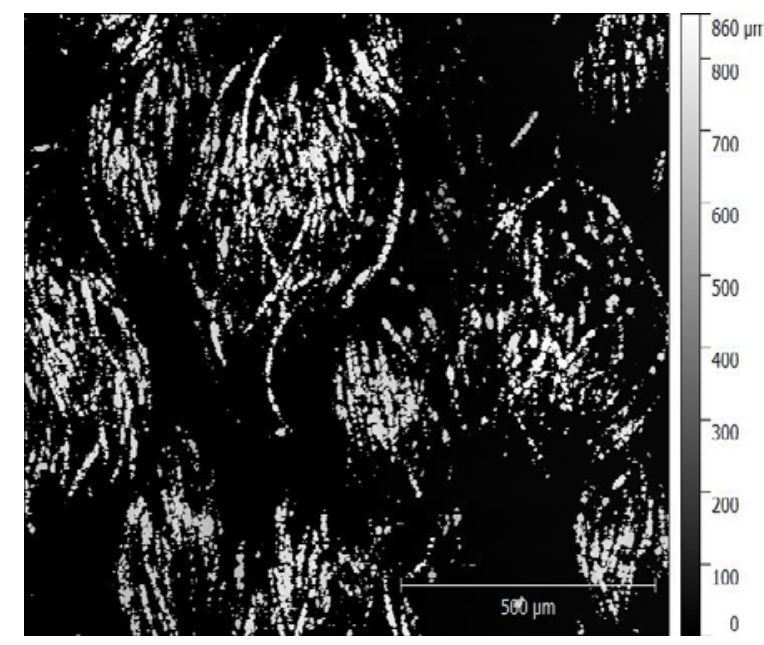

Ag Coated Nylon Jersey Knit (5x)

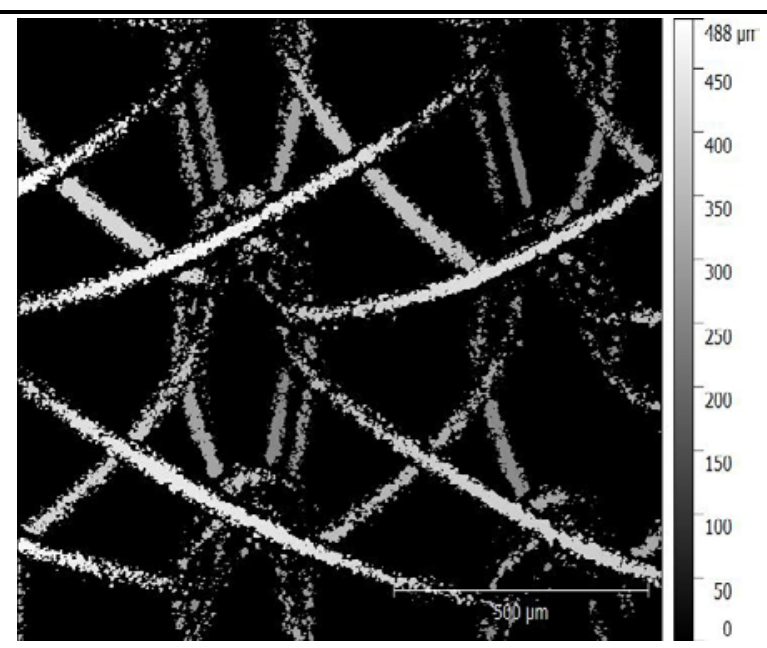

Ag Coated Nylon Tricot Knit (5x)

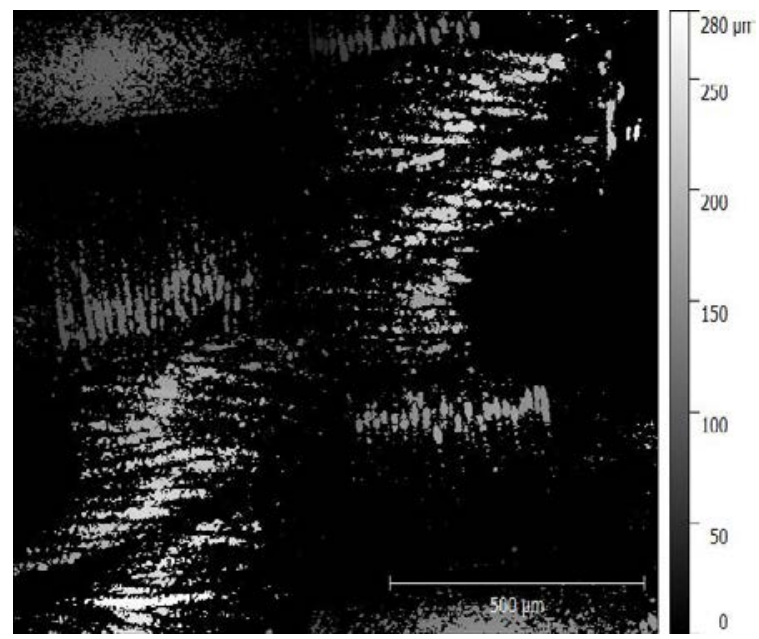

Ag Coated Nylon Hook (5x)

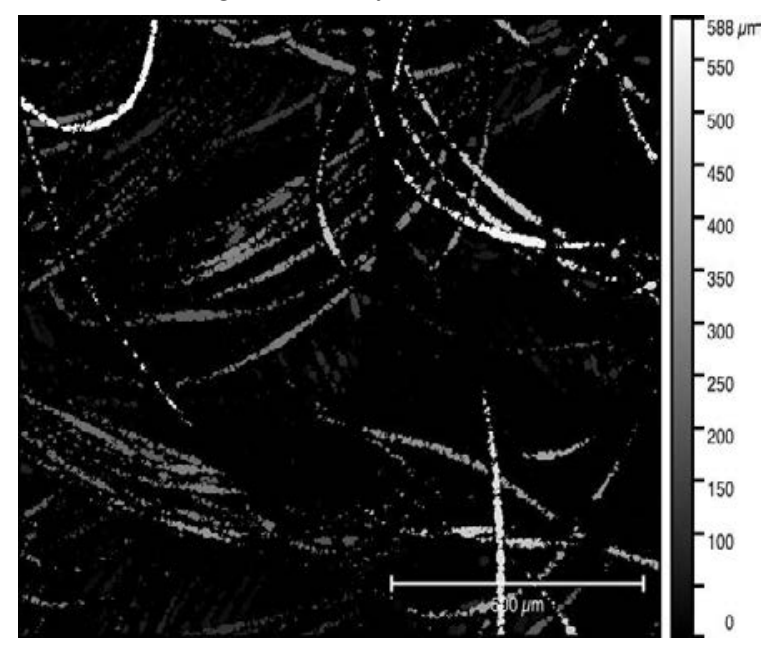

Ag Coated Continuous Nylon Loop (5x) 




Ag Coated Nylon Knit, 48x21 (5x)

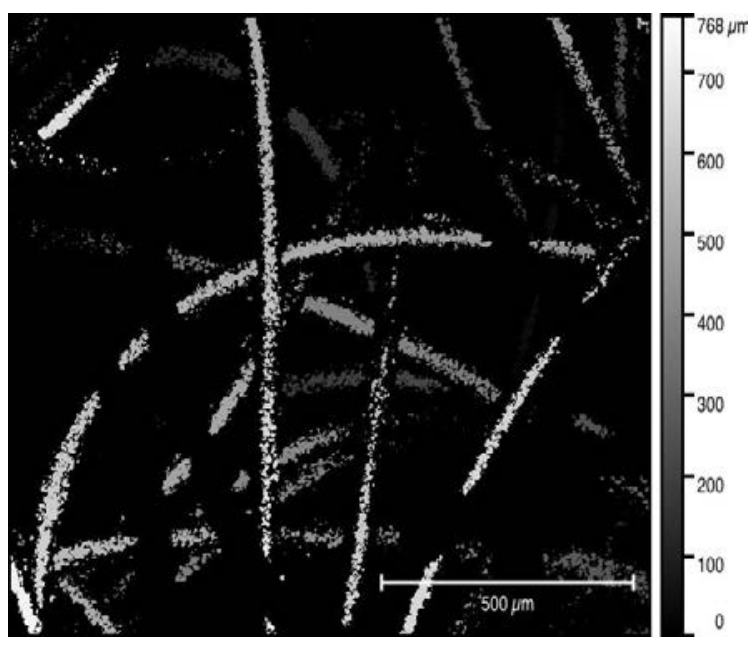

Ag Coated Nylon Loop (5x)

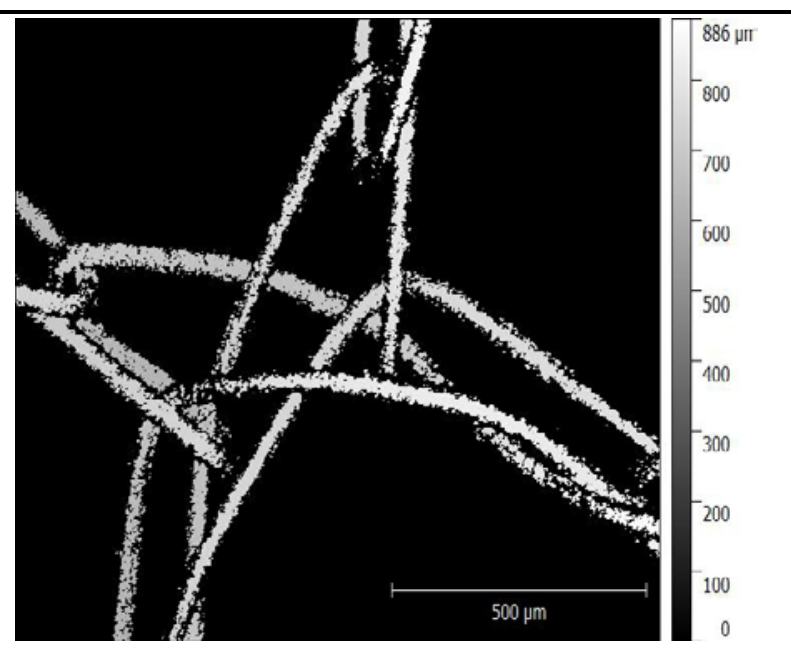

Ag Coated Nylon Knit, 13x26 (5x)

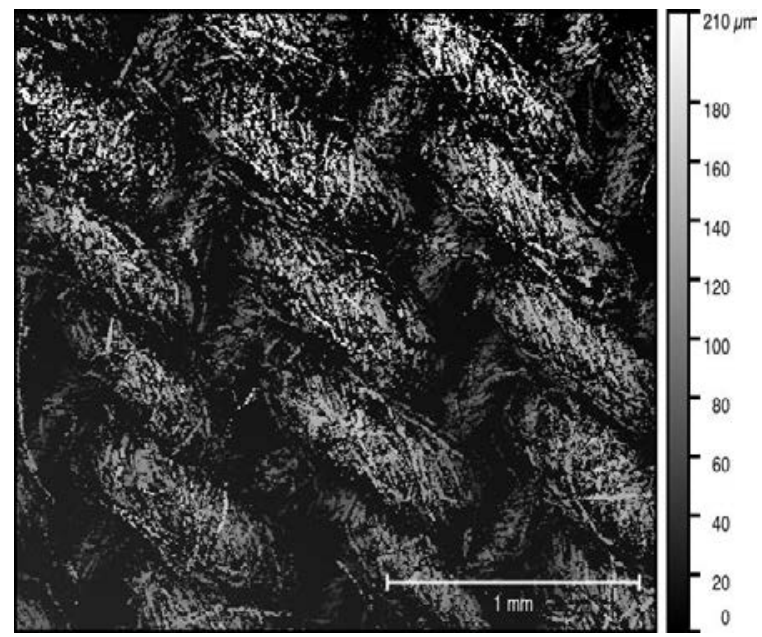

TexWipe 304 (5x) 


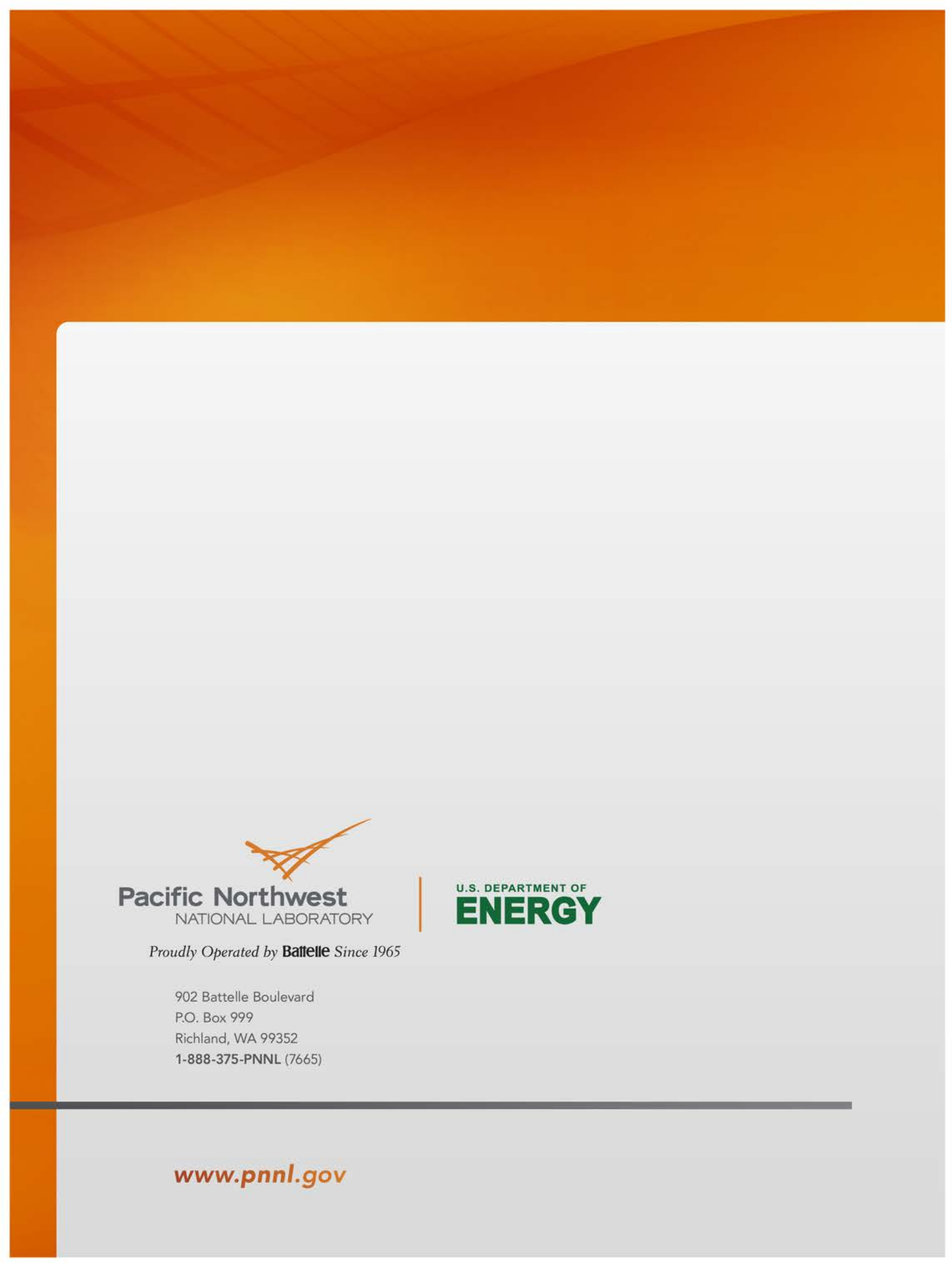

